\title{
Composites of functional polymers: Toward physical intelligence using flexible and soft materials
}

\author{
Michael J. Ford ${ }^{1, a)}\left(\mathbb{D}\right.$, Yunsilk Ohm², Keene Chin ${ }^{3}$, Carmel Majidii ${ }^{2,3}$ \\ ${ }^{1}$ Materials Science Division, Lawrence Livermore National Laboratory, Livermore, CA 94551, USA \\ ${ }^{2}$ Department of Mechanical Engineering, Carnegie Mellon University, Pittsburgh, PA 15213, USA \\ ${ }^{3}$ Robotics Institute, Carnegie Mellon University, Pittsburgh, PA 15213, USA \\ a) Address all correspondence to this author. e-mail: mjford0790@gmail.com \\ Received: 30 June 2021; accepted: 2 September 2021; published online: 19 October 2021
}

Materials that can assist with perception and responsivity of an engineered machine are said to promote physical intelligence. Physical intelligence may be important for flexible and soft materials that will be used in applications like soft robotics, wearable computers, and healthcare. These applications require stimuli responsivity, sensing, and actuation that allow a machine to perceive and react to its environment. The development of materials that exhibit some form of physical intelligence has relied on functional polymers and composites that contain these polymers. This review will focus on composites of functional polymers that display physical intelligence by assisting with perception, responsivity, or by off-loading computation. Composites of liquid crystal elastomers, shape-memory polymers, hydrogels, self-healing materials, and transient materials and their functionalities are examined with a viewpoint that considers physical intelligence.

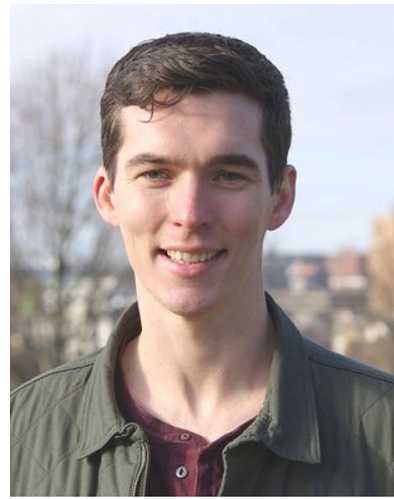

Michael J. Ford
Michael J. Ford is a postdoctoral researcher at Lawrence Livermore National Laboratory (LLNL). He earned his PhD in Materials from the University of California, Santa Barbara, where he studied polymer semiconductors. His position at LLNL follows a postdoctoral position in the Soft Machines Lab at Carnegie Mellon University. Under the direction of Prof. Carmel Majidi, Michael focused on the synthesis and characterization of new multifunctional materials for soft machines. At LLNL, Michael is a member of the High Performance Materials group and the Abilities Champions Employee Resource Group.

\section{Introduction}

Human-machine interactions will be ubiquitous in future technologies like soft robotics and healthcare. Materials that will be used for these technologies will need to be mechanically compliant and elastic to accommodate human-machine interactions. In addition, efficient soft machines will rely on materials that exhibit functionality beyond serving as structural elements and packaging for actuators and/or sensors. Soft machines can be built using functional polymers (e.g., shape-morphing materials) and composites that incorporate functional polymers. The idea of physical intelligencewhere functionality is encoded into a physical entity rather than enabled by a traditional computer-has been invoked to describe this emerging standard for materials and machines. 
A major motivator for the continued development of physical intelligence in compliant materials is that these materials will reduce or eliminate the computation that is handled by on-board electronics. By reducing computational complexity, machines that are fabricated using these intelligent materials can operate more readily in unstructured, real-world environments, which are inherently difficult for machines to navigate autonomously [1].

An entity can be described as intelligent if it complies with a set of rules and can use those rules in a diverse way. Researchers who study intelligence in engineered systems may divide their efforts into (i) embodied intelligence, where intelligence requires a body that assists with complying to the rules of its environment and exploits those rules to exhibit diverse behavior; (ii) computational intelligence, where a computer can learn a specific task from data; and (iii) artificial intelligence (AI), where a system algorithmically emulates the decision-making ability of a human expert [2]. Physical intelligence is related to these subsets of intelligence. For example, a material that exhibits physical intelligence may or may not enable embodied intelligence, depending on the machine. Physical intelligence can manifest as embedded perception and responsivity [1] (i.e., "the material is the machine" $[3,4]$ ) or can facilitate embodied intelligence by off-loading computation to the structure of the machine [1].

A typical example that illustrates off-loading of computational complexity involves the function of tendons in an organism's leg. Rather than requiring control effort to return the leg to its original position, the elasticity of the collagen fibers that comprise the tendons performs the work. The elasticity of materials like collagen fibers is not traditionally viewed as "intelligent," but the morphology of the body in combination with the physical properties of its materials create a replacement for active computational control. Illustrative examples of machines that exhibit a similar form of intelligence include a robotic gripper based on jamming of granular material [5] and a pneumatic crawler that navigates and grows via eversion [6].

In the case of the robotic gripper, which is composed of a balloon filled with a granular material, the granular material conforms to objects that it is pressed against. Then, the balloon is evacuated, and the granular material jams to grip the object that requires lifting (Fig. 1a). The gripper does not rely on sensors to determine the shape/orientation of the object, thus reducing computational complexity. For the crawler that grows via eversion, its pneumatic nature permits it to distribute pressure throughout its body. Therefore, it can squeeze through small gaps and change shape without requiring sensors or actuators to measure the size of the gap. In the case of the gripper, physical intelligence permits interaction with objects in its environment; for the crawler, physical intelligence facilitates movement through the environment. These simple examples illustrate some of the goals that researchers should consider when designing new materials with physical and/or embodied intelligence in mind; however, the future of multifunctional materials research will require developments that are much more complex and more tightly integrated.

We can discuss the complex functionalities that new materials will need so that machines can operate alongside humans in real-world environments by comparing modern machines to prokaryotes, fungi, algae, plants, and other simple organisms. For example, many plants are capable of regenerating tissue or organs. Damage to plant tissue can induce a hormonal response that triggers this regeneration (Fig. 1b) [7]. A superficial view of intelligence (i.e., without considering physical/embodied intelligence) may necessitate a brain or central processing unit to exhibit intelligence; however, plants that regenerate tissue/ organs do not rely on any centralized control unit. The sensing and responsivity of a plant that regenerates tissue/organs are enviable when considering the current state-of-the-art of materials and machines. For a robotic arm, if connection to the gripper of the arm is severed, the arm would not be fully functional until an engineer performed repairs. One improvement would be to include self-healing materials so that the engineer could simply reconnect the severed parts to complete the repairs. Ideally, the machine would sense that the connection was damaged, actuate the severed ends until they were rejoined, and then induce self-healing (Fig. 1c). An approach that considers physical intelligence to achieve this responsive self-healing would involve locally structuring the material to sense and respond to the damage without input from a central processing unit.

To fabricate compliant materials that can sense and/or respond to stimuli, we will focus on composites that use functional polymers. This focus allows us to narrow our scope in describing efforts that exemplify physical intelligence [1]. Remarkable progress in polymer chemistry has enabled new functionalities like actuation, conductivity, stimuli-responsiveness, self-healing, and reprocessability. Materials with these functionalities are contrasted from the "off-the-shelf" products (e.g., polysiloxanes) that are commercially available [8]. As these synthetic techniques are further refined in order to simplify reaction schemes and incorporate environmentally friendly precursors $[9,10]$, researchers have adopted these techniques to design composites that incorporate a functional polymer along with a filler. Many precursors are commercially available at sufficient purity, and polymers can be synthesized without specialized requirements like oxygen-free environments, which means non-chemists can more readily synthesize functional polymers and related composites [8]. Thus, the first portion of this review will cover composites that include a functional polymer matrix. Following, we will discuss our outlook on the future of materials with physical intelligence. We review these multifunctional materials through a lens that considers physical intelligence. This perspective will be useful as technologies that focus on 

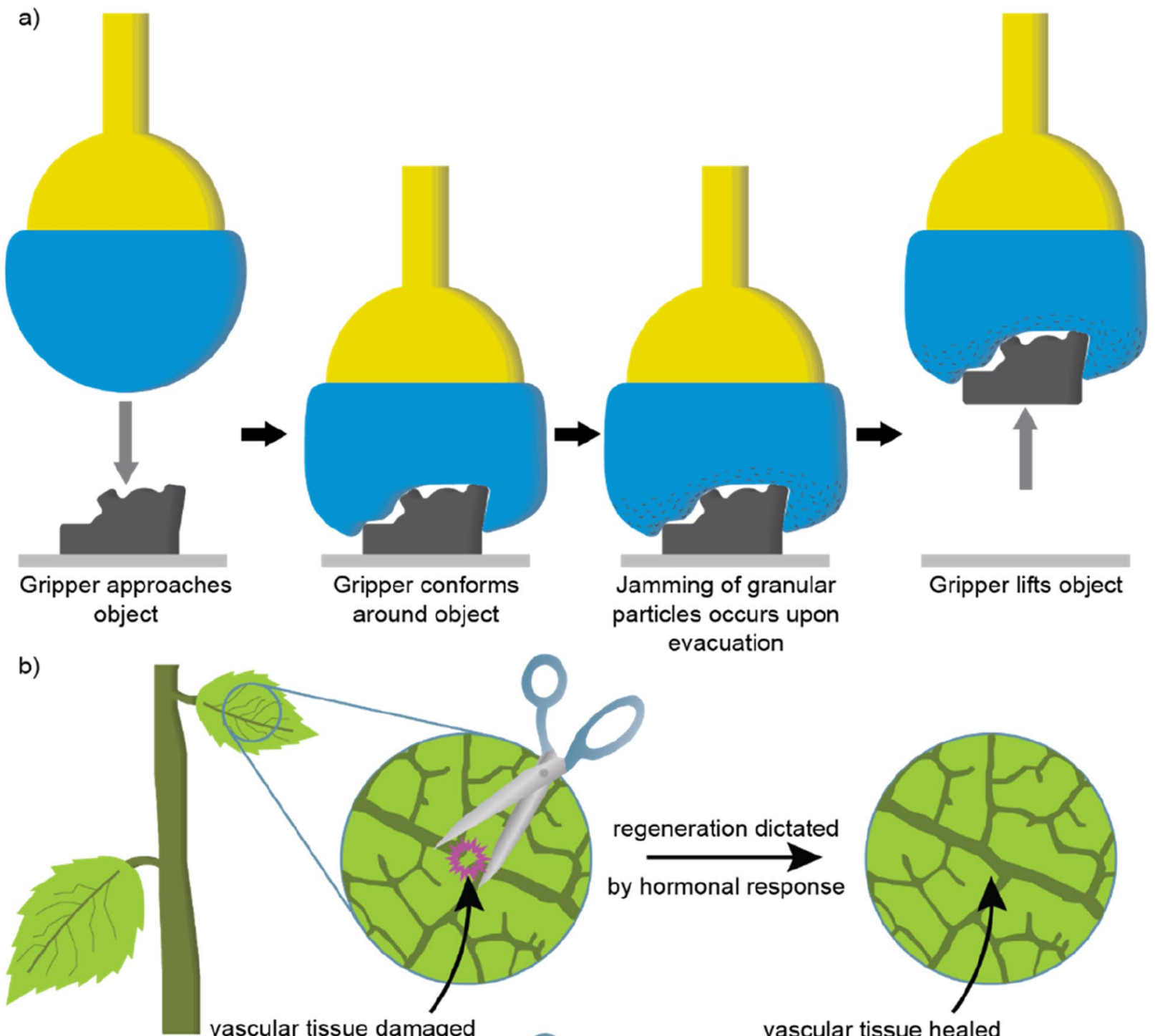

c)

vascular tissue damaged evacuation

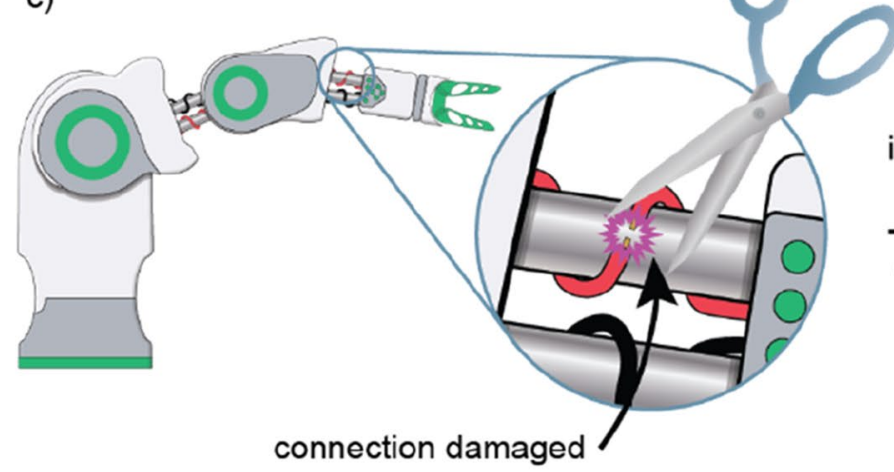

i) engineer repairs connection OR ii) responsive
self-healing

connection healed/repaired

vascular tissue healed

Figure 1: (a) A soft gripper can conform around an object without relying on sensors due to its composition, as shown in the illustration. This example highlights embodied intelligence, where low-level sensing is eliminated due to the compliance of the material. (b) A plant exhibits functions that can be viewed as intelligent even though it does not possess a brain, as shown in this illustration. A plant can experience tissue damage and heal due to hormonal responses that are embedded in the structural hierarchy of the plant. (c) Materials and machines strive to mimic some of the intelligent functionality, like self-healing, that is observed in simple organisms as shown in this illustration of a robotic arm that is damaged and healed. A responsive system that exhibits physical intelligence could heal without human intervention. 
human-machine interaction demand that compliant materials exhibit multifunctionality.

\section{Composites with functional polymers}

\section{Liquid crystal elastomers}

Liquid crystal elastomers (LCEs) are polymer networks that include a liquid crystal moiety that enables shape morphing. The liquid crystal units form ordered domains that are anisotropic. These domains transition between a disordered state and an isotropic state, typically due to a thermal transition or a response of a photoswitchable chemical unit. This transition results in a macroscopic shape change [11]. The shape change can manifest as simple linear motion, acting as an artificial muscle, or can be programmed to undergo more complicated shape transformation (e.g., a flat sheet at higher temperatures that forms features with Gaussian curvature when cooled) [12, 13]. One way that complex shape changes can be achieved is through additive manufacturing, which imposes a shear that aligns the liquid crystal domains [14-16]. Alternatively, complex shape changes that are not limited to flat sheets can be formed by aligning domains in the presence of a magnetic field during processing [17-19]. The ability to change to/from complex shapes is a key benefit of using LCEs for actuation. In contrast, systems of micromotors and controllers would be prohibitively complex to achieve the complicated embodiments that have been realized with LCEs.

\section{LCEs with photoresponsivity}

LCEs can be engineered to reversibly change shape in response to light-based stimulation. For example, photothermal actuation propelled a $14 \mathrm{~mm}$ strip of LCE that was programmed to undergo motion that resembled an inchworm. The crawler incorporated a dye with a peak absorption of $502 \mathrm{~nm}$, which was touted as an improvement for human-machine interactions relative to chromophores that absorb UV light or that require high light intensities for actuation [20]. A similar photoresponsive embodiment incorporated a liquid crystal solvent along with the dye and LCE matrix. The liquid crystal solvent reduced the transition temperature to permit actuation in water [21]. As with similar light-driven swimmers that had been previously reported [22], motion could be controlled by light. In this case, the swimmer would move toward the light as it flashed on and off (Fig. 2a) [21].

A similar mechanism was used to create an "optical flytrap," which resembled the action of a Venus flytrap. An optical fiber was attached to an LCE. When an object entered the field of view of the optical fiber, light was reflected and scattered. This light induced a shape change in the LCE, allowing it to grasp objects (Fig. 2b) [23]. In the view of physical intelligence, these embodiments do not require sensors that would trigger actuation. Instead, the actuator and sensor are built into one material. As a particularly compelling example of physical intelligence, the flytrap displays reactivity to its local environment, closing around an object only when the object is proximate to the gripper without a central processor [23].

\section{Joule heating of LCEs}

There may be tradeoffs that favor other methods of actuation instead of photoresponsive actuation of LCEs. For example, since organic dyes absorb light so strongly, photoresponsive actuation is typically limited to thin films (ca. $<1 \mathrm{~mm}$ ). One alternative method to actuate LCEs relies on Joule heating. To achieve Joule heating, common procedures either incorporate conductive particles during processing of the LCE or laminate a conductive film atop a LCE sheet [24]. However, the rigidity of conductive materials like carbon black restricts the actuation of LCEs at the loadings required to achieve conductivity. Serpentine patterns of conductive wires have been used to mitigate this issue. In one example that used serpentine wires, He et al. fabricated the LCE into a tubular actuator with three separate wires, enabling complex actuation in three dimensions. The geometry was leveraged to achieve complex actuation-in addition to a walker that moved through its environment without a tether (Fig. 2c)-without requiring difficult techniques to process the LCE actuator (e.g., those that require high magnetic fields) [25]. Future work that utilizes similar actuators/walkers as this work could show interesting locomotion by incorporating LCEs that actuate at different temperatures [26]. This strategy follows principles that are considered for embodied intelligence, where the morphology of the agent should be carefully considered to minimize computational effort.

Another method to eliminate the detrimental effect that rigid conductive fillers [27] can have on the LCE actuation is to use a deformable conductive filler instead. We developed a LCE composite that incorporated particles of a gallium-based liquid metal (LM); when large enough particles are used, the deformability of the LM particles did not restrict actuation (Fig. 2d) [28, 29]. These composites could conduct heat more efficiently than unfilled materials, allowing for efficient heating of thick specimens (e.g., $3 \mathrm{~mm}$ or more). Interfacial effects are significant when smaller LM particles are used (on the order of $10 \mu \mathrm{m}$ or less), and actuation strain and stress can be tuned. One limitation of Joule heating is that cooling to room temperature is slow, but these actuators can operate at high actuation frequencies with large actuation strains (e.g., $2 \mathrm{~Hz}, 15.5 \%$ ) in a convective fluid, like water [29]. As with all Joule heating, input power for these actuators is high due to heat wasted to the ambient environment. 

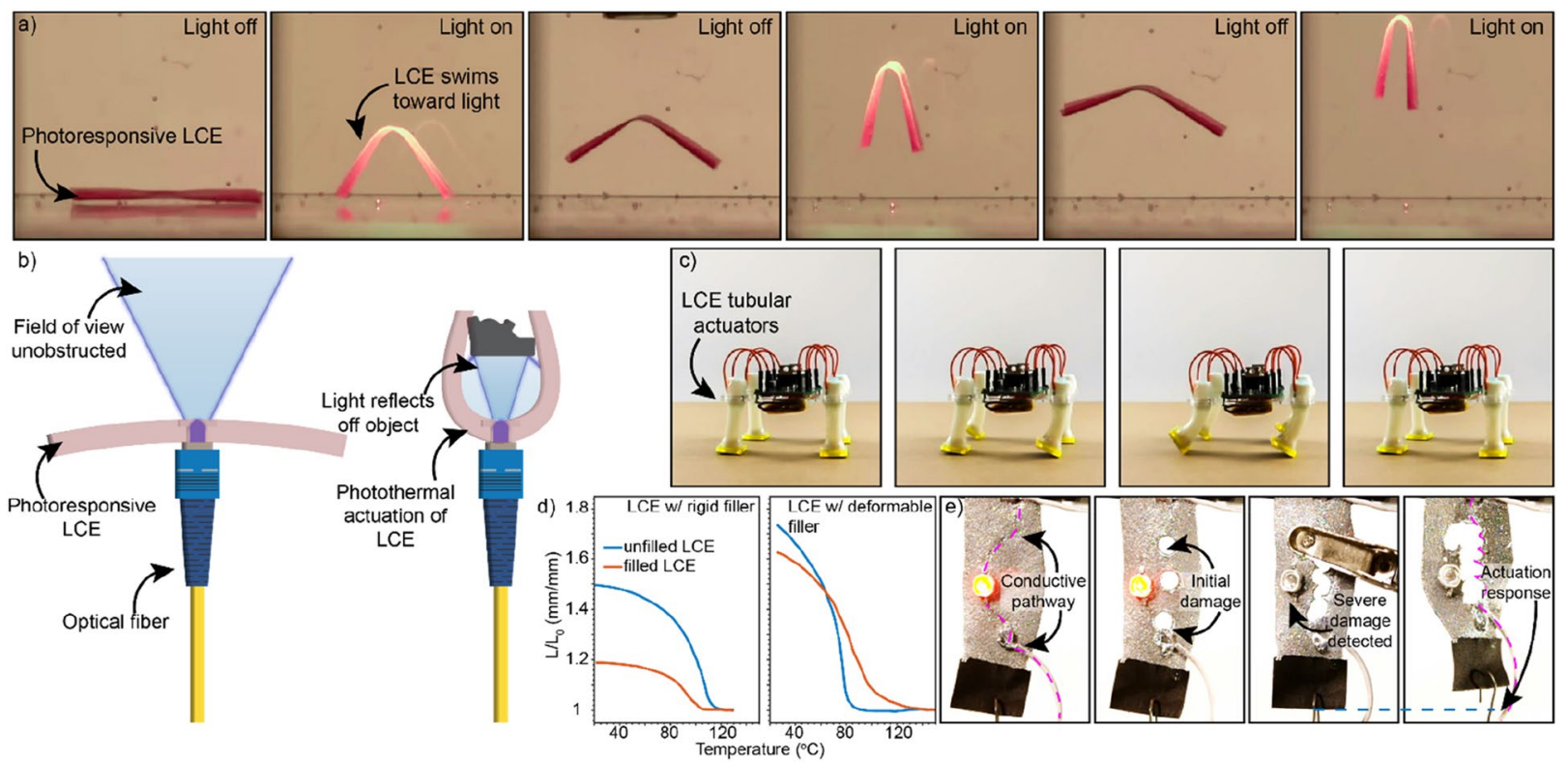

Figure 2: (a) A photoresponsive LCE can swim toward light, as shown in these sequential photographs. The LCE bends upon photothermal heating and can swim upward as the light flashes on and off. For scale, the dimensions of the swimmer are $16 \mathrm{~mm} \times 3 \mathrm{~mm} \times 0.1 \mathrm{~mm}$. Reproduced with permission from Ref. 21. Copyright 2020, National Academy of Sciences. (b) A photoresponsive LCE coupled with an optical fiber forms a gripper that grips without traditional sensors, as shown in this illustration, which is sketched based on Ref. 23. Without an object obstructing the light from the optical fiber, the LCE remains open (left). When an object is close enough to the end of the optical fiber, light is reflected toward the LCE, resulting in actuation (right). (c) LCE tubular actuators can power an untethered machine that walks using Joule heating, as shown in these sequential photographs of a single gait of the machine. The LCE actuators use serpentine wires to avoid restricting motion of the LCEs. For scale, the tubular actuators are approximately $0.7 \mathrm{~cm}$ in diameter. Reproduced with permission from Ref. 25. Without an object obstructing the light from the optical fiber, the LCE remains open (left). When an object is close enough to the end of the optical fiber, light is reflected toward the LCE, resulting in actuation (right). (c) LCE tubular actuators can power an untethered machine that walks using Joule heating, as shown in these sequential photographs of a single gait of the machine. The LCE actuators use serpentine wires to avoid restricting motion of the LCEs. For scale, the tubular actuators are approximately $0.7 \mathrm{~cm}$ in diameter. Reproduced with permission from Ref. 25. Copyright 2019, American Association for the Advancement of Science. (d) Rigid fillers like carbon black restrict LCE motion (left) to a greater extent than deformable fillers like liquid metal (right), as shown in these plots of the normalized change in length ( $L$ length at a given temperature; $L_{0}$ length in the isotropic state) vs. temperature. For large actuation strokes, $L / L_{0}$ should be maximized. For the LCE filled with a rigid filler, about 4 vol\% carbon black was used. For the LCE filled with a deformable filler, 50 vol\% eutectic gallium indium was used. Data were taken from Refs. 28 and 29. (e) An LCE actuator can sense and respond to damage by rerouting conductivity, as shown in these sequential photographs. The LCE composite is filled with liquid metal, which can be selectively patterned to be conductive in certain regions. In the first photograph, the LCE composite powers an LED with the conductive pathway highlighted in pink. The LED remains on after initial damage (second photograph) but significant damage reroutes conductivity, which turns off the LED (third photograph). The conductivity that was rerouted powers actuation through Joule heating (fourth photograph). The new route of conductivity is highlighted in pink. For scale, the LCE composite is about $3 \mathrm{~cm}$ wide. Reproduced with permission from Ref. 29. Copyright 2019, National Academy of Sciences.

We attempted to demonstrate an example of physical intelligence using these LM-LCE actuators. One attribute of composites that incorporate LM particles is that the composite is typically not electrically conductive until droplets are forced to coalesce (e.g., by mechanical sintering) [30]. Thus, it is possible to pattern the composite so that only some regions are conductive. Mechanical damage can also re-route conductivity, allowing for self-healing and localized damage detection [31, 32]. For our demonstration, conductive traces in the LM-LCE composite were patterned into a circuit that powered an LED. Damage occurred in a specific region of the composite, which rerouted the flow of current and activated Joule heating. The LM-LCE could respond to the damage by actuating (Fig. 2e). No control mechanism was used, i.e., the input voltage was not modified after it was turned on to power the LED [28]. However, we note that this example of physical intelligence is highly predetermined and only worked in the precise scenario that was constructed. The damaged region that triggered a reaction was too localized, and a distributed response that can also identify where damage occurred would be more impactful. Progress in physical intelligence will require similar responsivity in lessthan-ideal situations.

Other examples of physical intelligence that utilized Joule heating of LCE actuators involved LM traces rather than LM particles. LM traces exhibit electromechanical coupling that is reliable and predictable [33]. Thus, the resistance can be used to measure the strain. For an LCE actuator that is patterned with and powered by LM traces, closed-loop control of actuation is possible. In this case, the LM acts as the heating element and sensor simultaneously [34]. More recently, others have leveraged 
co-extrusion to integrate a liquid metal core into LCE strands. This method of processing provides a straightforward route to seal the LM from the ambient environment, which also reduces waste heat, while mitigating the electronic breakdown that was problematic in the previous LCE actuator that used LM traces. These so-called innervated LCEs could be printed to morph into complex shapes, and closed-loop control that relied on the change in resistance during heating was also demonstrated [35].

Similarly, other groups have printed LM traces atop LCEs and supplied a constant current. As the resistance of the LM increased due to the shape morphing of the LCE, a blue LED that was connected to the circuit became dimmer until it turned off. As a more useful demonstration of physical intelligence, the researchers designed circuits of LM traces that would respond to pressure or strain. For example, when constant current was applied by a power source, the resistance would increase when a pressure was applied, resulting in an increase in applied power that led to actuation. To leverage this sensing, a gripper was fabricated that responded to stretch. When the gripper was stretched, resistance increased, and the gripper opened. Upon relaxation of the applied strain, the gripper cooled and closed [36]. Such responsivity to the actuator's environment without the use of sensors or a central control unit is an impressive display of physical intelligence.

\section{LCEs with magnetic responsivity}

LCEs can also be paired with magnetic particles to impart an additional responsivity beyond what has been previously discussed. In early reports of these LCE composites, magnetic particles converted energy from an AC magnetic field into heat, which induced a phase transition in the LCE [37]. Another report introduced a novel functionality for LCE composites: magnetic memory that was rewritable. Anisotropic magnetic nanoparticles were oriented upon mechanical deformation of the LCE composite, which represents a stored state. When heating above the transition temperature of the LCE, the orientation of the magnetic nanoparticles becomes isotropic, which represents erasure of the stored state [38]. While memory is important for physical intelligence, it remains a challenge to figure out how this embodiment could be implemented and how the stored memory could be accessed and utilized by an intelligent machine.

LCE composites that contain magnetic particles can also exhibit responsivity that depends on the local environment. LCE composites that contain magnetic particles can exhibit responsivity in a magnetic field, which can allow for an additional mode of control and actuation. LCE composites with ferromagnetic microparticles were synthesized and exhibited complex shape deformations that are typical for unfilled LCEs. For example, a rectangular sheet could morph into a helical shape.
With magnetic particles added, the sheet would morph differently depending on magnetic programming and the applied field. At room temperature in air, the LCE composite walked when using a sinusoidal magnetization profile in the presence of an oscillating magnetic field. The composite walked to the edge of a substrate and then fell into a heated bath of glycerol. The thermal energy from the bath induced a shape change, and the composite form a helical shape. The composite could then swim upward or downward after the magnetic field was modified [39]. This example of physical intelligence represents an important step towards adaptability to local environments.

\section{Summary and future directions}

LCE composites are exemplary of the progress toward materials with physical intelligence. The examples outlined in this review are superficial in scope compared to the potential for LCEs. LCEs exhibit functionalities beyond what is covered in this review (e.g., dynamic/structural color change [40, 41] and mechanical dissipation [42]). Additionally, LCE chemistry has benefited from the progress of polymer chemistry $[9,43]$, which has enabled exchangeable covalent linkages [44], printable inks with tunable transition temperatures [26], and actuation blocking stress above $1 \mathrm{MPa}$ (with work densities that range from about $30 \mathrm{~kJ} \mathrm{~m}^{-3}$ up to about $1200 \mathrm{~kJ} \mathrm{~m}^{-3}$ ) [24, 45], among other features $[46,47]$. It is exciting to imagine the progress that will emerge as a result of these continued developments.

\section{Shape-memory polymers}

Like LCEs, shape-memory polymers (SMPs) provide the capability to change shape with large recoverable strains using a compliant material [48]. For SMPs, the mechanism for shape changing relies on a phase transition (e.g., the formation of crystalline domains or a glass transition). Synthesis of SMPs determines the permanent shape. The shape from which the material cycles back and forth to the permanent shape is called the programmed shape. In many cases, the programmed shape is produced by deforming the material above the melting point, followed by cooling to form crystalline domains. The crystalline domains lock in the programmed shape, and entropy drives the return to the permanent shape upon melting under stress-free conditions. The most ubiquitous class of SMPs is heat-shrink tubing and film, which exhibit a one-way shape-memory effect, transforming from the programmed shape to the permanent shape.

There is no intrinsic mechanism for SMPs with one-way shape memory to return to their programmed shape [49]. To actuate back and forth between two shapes, SMPs are composed of either two crystallites with distinct melting temperatures or a melting temperature that is broad $[49,50]$. Alternatively, twoway shape memory is possible for a SMP that has a single melting transition if the SMP is under a constant stress [51]. For 
materials with physical intelligence, two-way shape memory broadens the responsivity and allows for repeatability of an intelligent response (i.e., actuation encoded in the material). An important feature of SMPs with two-way shape memory is the ability to reprogram the reversible shape morphing. Once synthesized, a SMP specimen could exhibit innumerable movements following reprogramming [49]. Additionally, SMPs with multiple melting temperatures or a melting temperature that is broad can be programmed to transform between multiple shapes (i.e., greater than two depending on the number of melting temperatures that can be accessed, which is also influenced by the cooling rate and applied deformation) [50,52].

\section{Joule heating of SMPs}

The strategies that have been used to activate SMP actuation are similar to those that have been used to activate LCE actuation. A recent review covers many of the embodiments of active actuation of SMPs [53]. For example, conductive fillers have been used for Joule heating of SMPs in various reports [54-57]. In one report, a combination of carbon black (CB) and carbon nanotubes (CNTs) operated in tandem to produce electrical conductivity in a SMP. The CNTs bridged aggregates of CB to form a conductive network [56]. As discussed with LCE composites, the conductive fillers permit Joule heating and sensing simultaneously. In one case using SMPs, the change of the resistance as temperature changed was characterized although a demonstration of physical intelligence was not developed [58].

Carbon-based fillers may result in SMP composites with resistivity that is too high for many practical uses. Therefore, conductivity was improved in a SMP composite by using CB and Ni powder. The Ni powder can be directed to assemble into chains in the presence of a magnetic field. For a SMP composite that contains $10 \mathrm{vol} \% \mathrm{Ni}$ powder that is aligned, shape recovery by Joule heating is possible when $6 \mathrm{~V}$ is applied. For a SMP composite that contains $10 \mathrm{vol} \% \mathrm{Ni}$ powder that is not aligned, that same input electrical power was not high enough to result in shape recovery [59]. Similarly, Wei et al. took advantage of the high aspect ratio of CNTs and coated them with Ag to increase conductivity. A SMP composite that included these Ag-coated CNTs could be printed (e.g., into a compliant gripper) [60]. These composite highlights how advancements in processing can bring about new functionalities (e.g., responsivity to electrical stimulation) that are important for physical intelligence.

\section{SMPs with photoresponsivity}

Photothermal responsivity is one common route to active actuation of SMPs [61, 62]. With photothermal responsivity, a SMP composite that is connected to a wire that is connected to a battery terminal can locally change shape to close an electrical loop [63]. This example of physical intelligence combines the actuator and trigger for actuation in one material while also demonstrating additional responsivity as the electrical loop is closed. In another example that was responsive to infrared (IR) light, Liu et al. printed black ink atop a SMP specimen. These materials were then used as hinges to transform flat sheets into $3 \mathrm{D}$ objects [64]. The $2 \mathrm{D}$ to $3 \mathrm{D}$ transformation is exemplary of a change in form factor that would be favorable for applications like remote exploration or healthcare. Considering the idea of physical intelligence, this use of IR-responsive hinges could be extended by incorporating additional responsivity into the walls of the $3 \mathrm{D}$ objects. Then the $2 \mathrm{D}$ and/or $3 \mathrm{D}$ shapes could transform in a predetermined manner, depending on the order that the material interacts with each stimulus. A step toward this integrated responsivity was achieved when the ability to create intricate, $3 \mathrm{D}$ objects was demonstrated. The fabrication of these $3 \mathrm{D}$ objects relied on a transfer printing process that involved pre-straining the substrate onto which a SMP was cast. These objects were varied and complex, and useful demonstrations that included a coil antenna and remote actuation of the 3D structures were demonstrated [65]. Alternatively, magnetic SMP composites were coupled with sensors that detected the magnetization state and could be used for control that triggered actuation. The location of the hinge could be programmed at will, and then shape recovery was triggered photothermally, allowing for complex folding and unfolding [66].

Photothermal responsivity can be combined with responsivity to a magnetic field to create one-way SMPs that are useful for applications like soft robotics. As mentioned, a drawback of one-way SMPs is that they no longer can change shape once recovery is completed. Liu et al. used microparticles that were magnetically and photothermally responsive. The photothermal response triggered the shape recovery in the SMP. Then, a magnetic response permitted reprogramming remotely, which was possible since the photothermal heating softened the composite. This combined use of SMPs and magnetic microparticles expands the functionality of these one-way SMPs.

The local heating that triggers shape deformation due to absorption of light can also trigger other functions. Zhang et al. used gold nanoparticles, which convert IR light to heat, as a functional filler in a SMP. This photothermal effect enables shape recovery that can be localized in the material, as observed in examples that were previously referenced. In addition, the photothermal effect can also heal the SMP composite. This healing occurred when crystallites melted, chains diffused, and recrystallization occurred near the fractured surfaces. Again, this healing can be localized to only heal regions where cracks are present. Interestingly, healing did not occur when heating using a $120^{\circ} \mathrm{C}$ heat source. The use of an IR laser was necessary for healing; the authors attributed this requirement to the fact that localized thermal expansion was crucial to proper healing. As a demonstration of responsivity, healing or shape recovery 
was executed in a sequential manner. A SMP composite was folded into a temporary shape, and two cracks were cut into the composite. One cut was healed first without any shape recovery, followed by partial recovery of the original shape. Then the second cut was healed, followed by permanent recovery [67]. This multifunctionality while using a single filler material is a keystone of the future of physical intelligence. For advancement in physical intelligence, we imagine materials that do not rely on remote control of the IR laser for self-healing and shape recovery. Instead, materials will detect that damage has occurred, heal the damage locally, and then deform into the desired shape.

\section{SMPs with radiofrequency responsivity}

As mentioned, SMPs are advantageous due to their ability to be programmed to transform between multiple shapes. Selective triggering of multiple shapes is also possible using radiofrequency [68] (RF) and molding discrete regions of dissimilar $\mathrm{SMP}$ composites. CNTs and $\mathrm{Fe}_{3} \mathrm{O}_{4}$ nanoparticles were incorporated into a SMP matrix. CNTs induce heat at $\mathrm{RF}=13.56 \mathrm{MHz}$ while $\mathrm{Fe}_{3} \mathrm{O}_{4}$ nanoparticles induce heat at $\mathrm{RF}=296 \mathrm{kHz}$. By molding three discrete regions (CNT-SMP, unfilled SMP, and $\mathrm{Fe}_{3} \mathrm{O}_{4}$ ) into a monolithic specimen, the authors demonstrated that the material could transform into multiple shapes dependent on the input RF (Fig. 3a) [69]. As discussed, multiresponsivity (e.g., specific frequency of a stimulus source) may be necessary for some applications of materials with physical intelligence.

\section{Temperature memory effect}

Another intriguing functionality of SMPs is a so-called temperature memory effect $[70,71]$. The temperature memory effect occurs for materials with a broad shape-memory transition. If the strain in the material is fixed while heating, a maximum in the measured stress during shape recovery would occur at the temperature at which the material was deformed (Fig. 3b). For a composite that contains CNTs, interactions between the CNTs and the matrix influence the local glass transition temperature. The temperature memory effect in this composite relies on the broadened glass transition temperature [72]. The temperature
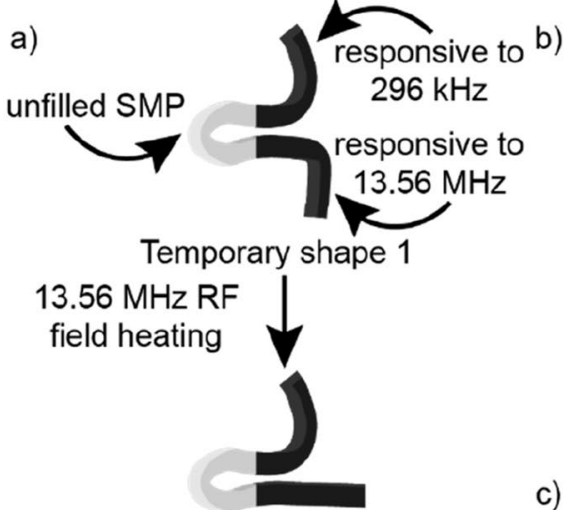

Temporary shape 2

$296 \mathrm{kHz}$ RF field heating

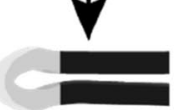

Temporary shape 3

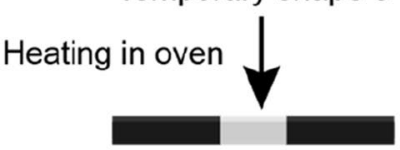

Permanent shape 4

c)
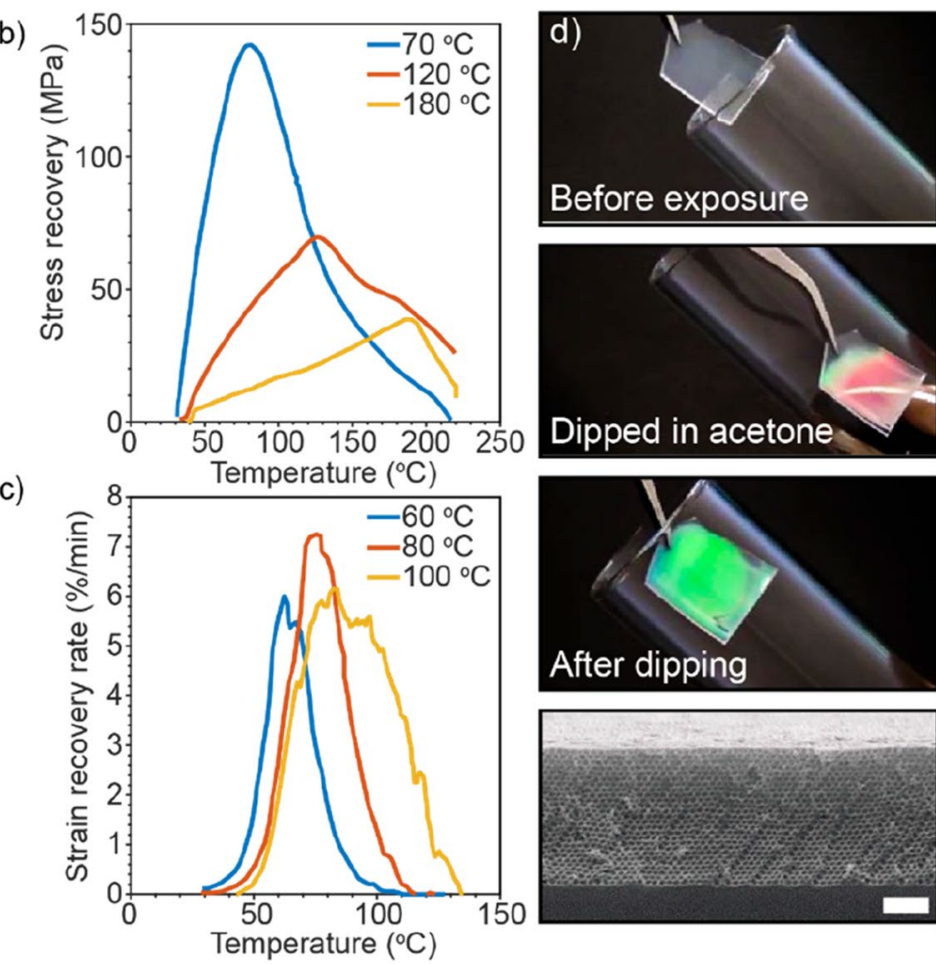
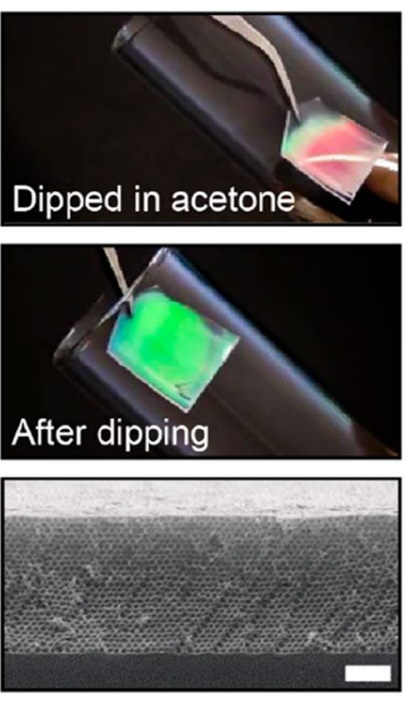

Figure 3: (a) Multiple fillers can be used to enable multiresponsivity (e.g., responsivity to different radiofrequencies (RFs)), as shown in this illustration, which is sketched based on Ref. 69. CNTs induce heat at $\mathrm{RF}=13.56 \mathrm{MHz}$ while $\mathrm{Fe}_{3} \mathrm{O}_{4}$ nanoparticles induce heat at $\mathrm{RF}=296 \mathrm{kHz}$. Transformation can occur between four shapes, depending on the input stimulus. Reproduced with permission from Ref. 69. Copyright 2011, John Wiley \& Sons. (b) A temperature memory effect manifests as a peak in the stress recovery as a function of temperature, as shown in these plots. Each trace represents different temperatures at which the SMP was programmed, which shifts the peak. Data were taken from Ref. 72. (c) The temperature memory effect can also be observed under isostress conditions by monitoring the strain recovery rate. Again, each trace represents different temperatures at which the SMP was programmed, which shifts the peak. Data were taken from Ref. 70. (d) Shape memory can be useful for signaling with changes in microstructure, as shown in these photographs and micrograph of a polymer that has a photonic structure (bottom image) that changes depending on solvent exposure. The scale bar in the micrograph represents $2 \mathrm{~cm}$. The width of the polymer specimen is approximately $1.3 \mathrm{~cm}$. Ref. 76 . Copyright 2015, John Wiley \& Sons. 
memory effect can also be observed when examining the rate of strain recovery, which also peaks at the deformation temperature (Fig. 3c) [70]. Even though the mechanism to gather information from such materials remains underexplored, materials capable of information storage is one need for materials that facilitate physical intelligence.

An interesting concern for SMPs that could be leveraged for physical intelligence involves overexposure to a stimulus. If a SMP that is programmed to actuate between two shapes is inadvertently heated above its programming temperature, then the memory of the programmed shapes will be erased. The SMP would remain in its permanent shape after it has cooled. This characteristic could be leveraged as a benefit rather than a detriment. The material could deactivate as a safety precaution to protect the machine. In addition, magnetic particles could be incorporated into the material so that the material can be reprogrammed remotely after the memory is erased [73].

\section{Summary and future directions}

Another feature of SMPs that may make them advantageous relative to other actuator materials like LCEs, for example, involves their chemical composition. SMPs are not limited to specific moieties and can be readily tuned to manifest new functionalities. The chemical composition of the SMP only needs units that can undergo a phase transition to bring about meaningful shape morphing. Thus, polyurethanes, polyisopropylene, copolymers of styrene and butadiene, polynorbornene, and other polymers have been explored as SMPs [74].

The chemical structure of the polymer can determine physical and chemical properties like mechanical strength, stimuli responsivity, and self-healing. For example, hydrogen bonding in polyurethane SMPs can be influenced by absorbed water, which influences recovery of the original shape. When three segments of a SMP were immersed in water for three different durations, their transition temperature varied and, thus, the temperature at which the shape recovered varied [75]. SMPs can also respond to solvent vapors. A photonic SMP structure that has dried following water exposure does not show any structural color. When solvent vapors like acetone or ethanol are absorbed, structural color that was programmed into the permanent shape recovers, and the SMP appears green. In this case, the responsivity is due to a structural feature of the SMP in conjunction with sensitivity to solvent vapors (Fig. 3d) [76]. The state of the SMP can also be indicated optically when the separation between plasmonic nanoparticles changes during deformation [77]. As mentioned, responsivity and sensitivity to a wide range of stimuli may be important for physical intelligence.

As researchers make advancements in polymer chemistry, new functionalities that are important for future applications like self-healing, responsivity, recyclability, and biodegradability [78-82] could be integrated into SMPs. One early example that highlighted the versatility of the chemical composition of SMPs incorporated light-responsive moieties into the network [61]. Additional fillers that introduce multiresponsivity could be used with a material that is intrinsically light responsive. Fillers also may improve actuation properties of SMPs. For example, as SMPs undergo a thermal transition, the mechanical properties may degrade. Additives that increase stiffness with temperature could counteract this effect. New materials could be amenable to additive manufacturing, which has been leveraged to produce intricate shapes along with photonic structures [83-85]. These advancements toward multifunctionality are important to bring about physical intelligence in SMPs. While original reports of SMPs focused on the versatility of the chemical structure, recent efforts are moving toward materials with physical intelligence, focusing on soft robotic, stretchable electronics, and similar applications [53, 73, 86-88]. Further advancements in physical intelligence in SMPs and related composites will rely on leveraging the versatility of the chemical composition of SMPs.

\section{Hydrogels}

Hydrogels have been useful as matrix materials for functional composites. These materials can intrinsically exhibit functionality besides actuation [89] that has been described so far including ionic conductivity [90], adhesion [91], reprocessability [92], and responsivity to temperature, $\mathrm{pH}$, ions, light, and chemicals [93]. Although monomers of common hydrogels can be toxic (e.g., acrylamide [94]), many hydrogels are considered biocompatible in terms of toxicity and mechanical properties [95]. Like SMPs, the topic of hydrogels covers a wide range of chemical structures, which bring about numerous functionalities [96]. Hydrogels can also be processed or synthesized to form double networks that improve properties like stretchability and toughness [97]. Hydrogels can be useful for applications like soft robotics and healthcare, which can require sensing, responsivity, and/or actuation [93, 98-100]. When processed as composites, hydrogels provide prime examples of materials with physical intelligence.

\section{Stimuli-responsive gelation of hydrogels}

One responsivity of hydrogels that has been explored is the change in gelation with temperature. Depending on the chemical structure of the hydrogel, gelation can occur as the temperature increases or decreases [96]. Zhang and Bellan capitalized on hydrogels that exhibit this temperature dependent gelation to create transient electronics. The hydrogels that were used differed in their lower critical solution temperature (LCST). A polymer and solvent form two phases above the LCST and are miscible below that temperature. Ag nanowires were used to form electrical circuits atop the gel structure when the material was above the LCST. A heat source was required to maintain 

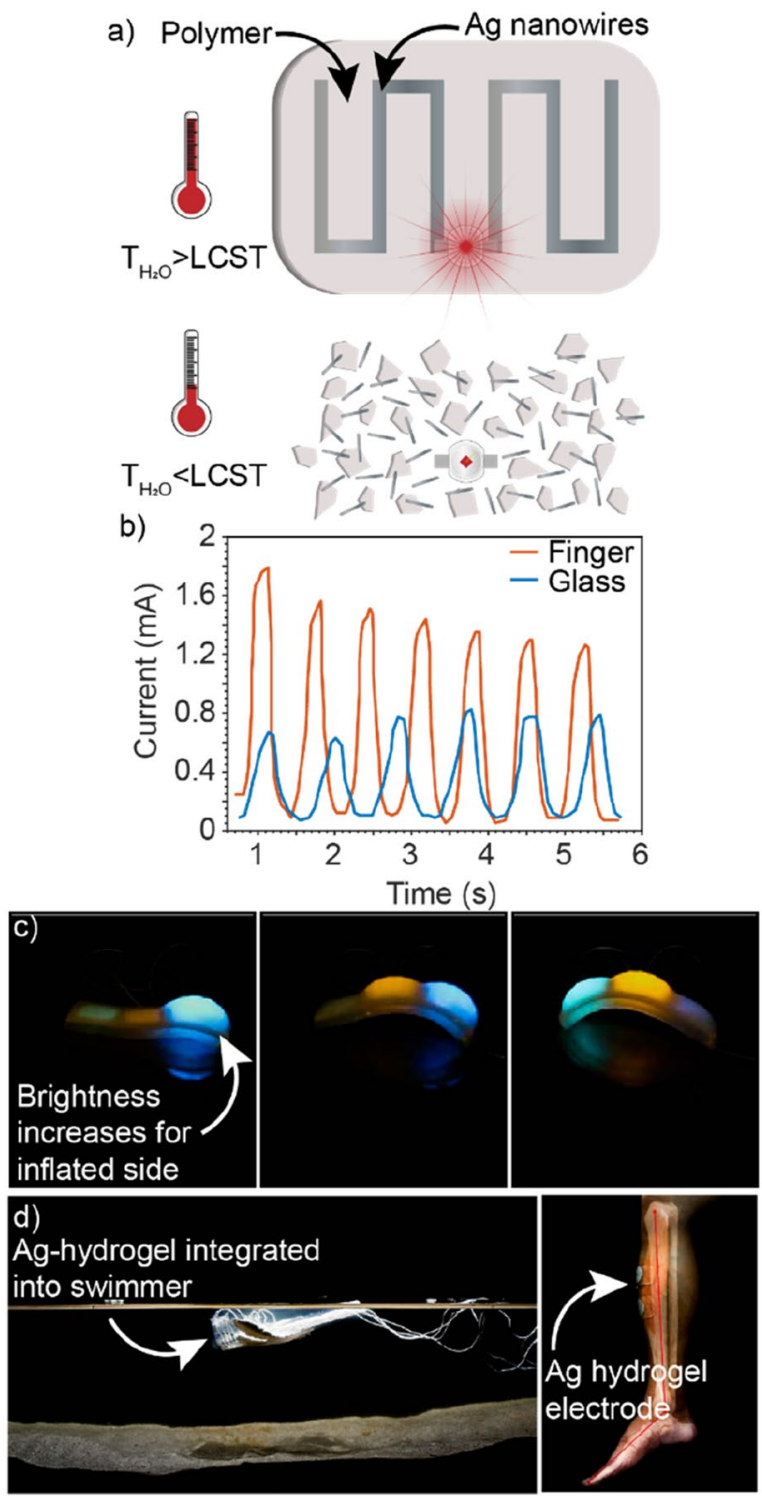

Figure 4: (a) A hydrogel composite maintains its shape above a certain temperature, while below that temperature the hydrogel breaks apart, as shown in the illustration, which is sketched based on Ref. 101. Using Ag nanowires, a transient electrical circuit can be created. (b) A warm object (finger) pressed against a hydrogel composite may exhibit a different response relative to when a cooler object (a glass sheet) is pressed, as shown in this plot of the current response vs. time. Data were taken from Ref. 102. (c) A soft robot can signal its current state due to changes in brightness upon inflation, as shown in these sequential photographs. For scale, the soft robot is $20 \mathrm{~cm}$ long. Reproduced with permission from Ref. 103. Copyright 2016, American Association for the Advancement of Science. (d) A hydrogel composite filled with Ag flakes relies on percolation induced during a drying process to achieve conductivity high enough to provide power to a soft swimmer (left) or to provide neuromuscular stimulation (right), as shown in these photographs. For scale, the body of the swimmer is about $24 \mathrm{~cm}$ long. Reproduced with permission from Ref. 105. Copyright 2021, Springer Nature.

the gel structure, which then was lost upon cooling. Upon cooling, the Ag nanowires were dispersed, destroying the electrical circuit (Fig. 4a). The authors demonstrated a transient circuit that turns an LED off when the temperature is lowered [101]. A challenge for transient materials for some applications involves reassembly of the materials at will. This transience in conductivity could also be coupled with non-transient materials to introduce functionality (e.g., coupled with an actuator that is physically restrained until the transient material is disassembled) and exhibit physical intelligence.

The LCST could also be used for sensing in conjunction with electrical conductivity. A two-dimensional transition metal carbide/nitride (MXene) was used to improve the conductivity of a hydrogel. Changes in current were monitored while the conductive hydrogel was heated. Heat from the palm of a hand could transition the composite through its LCST, resulting in a change in the current of the electrically conductive hydrogel composite. An intriguing attribute of the composite that relates to physical intelligence was the ability to distinguish between two different objects. When a warm object (a human finger) compressed the composite, the current increased to a higher value than when a cooler object (a glass sheet at room temperature) compressed the composite. The increase in current upon compression was due to two factors. The current increased due to volume changes as the temperature increased, and the current increased due to compression that increased interparticle contact of the fillers (Fig. 4b) [102]. This ability to distinguish different objects based on multiple attributes of the object is an advancement in physical intelligence. However, it is not clear whether the authors controlled for variations in compressive force that may have influenced the measurement. In addition to controlling for compression vs. temperature, future studies may attempt to create a material that can deconvolute increases in current due to compression and temperature. Either way, the volume change due to the LCST is an important feature of hydrogels. Similar changes in volume due to gelation can also occur due to changes in $\mathrm{pH}$, light, and chemical environment. This responsivity to multiple stimuli allows for several methods of actuation and sensing, which are important for physical intelligence [100].

\section{Ionic conductivity of hydrogels}

The ionic conductivity of hydrogels has also been leveraged for physical intelligence [90]. Due to this ionic conductivity, hydrogels are promising as transparent electrodes. In one example, a multi-layer structure that consisted of an ionically conductive hydrogel and an electroluminescent dielectric layer was produced. Pneumatic chambers were incorporated into the structure. Hydrogel layers served as the electrodes for electroluminescence and were transparent to allow light to transmit. The intensity of electroluminescence changed as the pneumatic chambers were filled due to a change in capacitance (Fig. 4c). Using a constant voltage, the state of the actuator could be 
recognized by observing the change in light intensity due to applied pneumatic pressure. This example demonstrates two principles of intelligence: proprioception and exteroception. The material could arguably be exhibiting proprioception as its capacitance and brightness changes when it moves to a new position. A central processing unit would likely be necessary, but if either of those properties can be known/measured by the material while it moves, then proprioception is exhibited. Regarding exteroception, the capacitance changes when an external pressure is applied (e.g., a finger touching the material). Thus, it has some way of understanding its environment [103]. However, it is unclear if it is possible to distinguish between these two events. That challenge is a common theme in many examples of multifunctional materials: can a material that can detect changes in its position and environment distinguish between the two when the stimuli occur simultaneously/randomly?

\section{Electrical conductivity of hydrogels}

Ionic conductivity may not be high enough for some applications; thus, additional conductive fillers could be introduced [104]. For example, we demonstrated that Ag flakes increase the conductivity of a hydrogel composite to allow for Joule heating. A partial drying and rehydration that reassembled the Ag flakes increased the conductivity from $0.1 \mathrm{~S} \mathrm{~cm}^{-1}$ to $370 \mathrm{~S} \mathrm{~cm}^{-1}$. Joule heating was then used to power a soft swimmer that resembled a sting ray. The composite was also used as an electrode for neuromuscular stimulation [105]. In another paper, hydrogel precursors were mixed with liquid metal droplets, which sedimented during curing to form a layer with high volume content of liquid metal. As we discussed, these liquid metal droplets are not conductive until a mechanical pressure coalesces the droplets. The nature of the hydrogel presents an interesting functionality: a conductive trace that is written into the hydrogel can be erased. The mechanism of erasure was that the surrounding hydrogel was locally swelled. It is possible that liquid metal that leaked during sintering also participated in the conductivity and that this liquid metal trace was disrupted during erasure. To erase conductivity, the composite was sprayed with water near the conductive traces, disrupting percolation of liquid metal [106]. The conductive traces could be rewritten, which could be useful for information storage or communication.

\section{Self-healing of hydrogels}

In addition to functionalities like stimuli responsivity and conductivity, hydrogels can also exhibit self-healing. A hydrogel that was capable of self-healing was combined with magnetic particles to impart responsivity to a magnetic field. A specimen was broken into three pieces and separated. A magnetic field was used to reassemble the three pieces into one piece, and after $10 \mathrm{~min}$, the specimen was healed. While this demonstration of utility required human intervention to reassemble the three pieces, it highlights the potential of remotely-and possibly autonomously-repairing a system that has experienced significant damage. This material also could also squeeze through a small gap in the presence of a magnetic field. It rearranged its shape without requiring sensors that would detect the gap that it was passing through, which demonstrates embodied intelligence [107]. This behavior may be a hinderance in applications like soft robotics where a gravitational field or continuous pressure may result in flow of the material over time. However, it may be useful for biomedical applications where injection and flow of a material are necessary.

\section{Summary and future directions}

Like SMPs, hydrogels offer a wide range of functionality due to the tunability of the chemical structure. When fillers are used to form composites, particle-matrix interactions can be studied and modified to bring about new functionalities. Hydrogels are also useful when considering biological interfaces; these materials have properties like adhesion, low toxicity, and softness that are desirable for biological interfaces [108]. As an example, adhesion could be tuned based on the chemical environment, permitting selective gripping of materials [109]. In addition, they can be used to sense and respond to biomolecules like glucose [110]. Hydrogels have been designed for both sensing and actuation. They have also been used to communicate signals [111] (e.g., optical or tactile) and as soft power sources [100]. Methods of communication and to provide power will be crucial for autonomous systems. Thus, the further development of hydrogels and related composites that exhibit physical intelligence will be important.

\section{Other functional polymers}

\section{Polymer composites with mechanical self-healing}

Soft materials that are susceptible to damage benefit from selfhealing, especially in unstructured environments. Common methods to synthesize self-healing materials include incorporating monomers that are released and react at the site of fracture and synthesizing networks that contain reversible covalent bonds. Many self-healing polymers that incorporate reversible covalent bonds into the polymer network require heating for full and/or fast healing [112]. Active heating and healing (e.g., at higher temperatures) can be achieved by a single material when self-healing polymers are processed as composites. For example, a composite incorporated magnetic fillers to induce selfhealing in an alternating magnetic field. In this case, the magnetic filler that was selected had a Curie temperature of $113^{\circ} \mathrm{C}$. Above that temperature, the magnetic fillers no longer exhibit magnetic susceptibility. In this case, the filler serves as a control 
mechanism to avoid overheating the composite, which could degrade the polymer [113]. In a soft machine, this composite would not require temperature sensors to avoid overheating. This idea was extended by changing the composition of the filler, which changed the Curie temperature [114]. Thus, using multiple fillers and directed assembly, localized regions of these composites could be heated to different temperatures under the same magnetic field.

\section{Polymer composites with electrical self-healing}

The development of materials for self-healing initially focused on restoring mechanical properties after healing. More recently, efforts have also highlighted the ability to restore other properties enabled by a composite filler, like electrical conductivity. For example, a self-healing polymer that encased a channel of liquid metal formed a conductive, stretchable wire. After the wire was damaged, the liquid metal formed an oxide layer upon exposure to air that prevented it from flowing out of the wire. When the two broken ends of the wire were placed in contact, the polymer healed. Electrical conductivity was restored due to the deformability of the liquid metal [115]. When processed as droplets in a self-healing matrix, liquid metal circuits can be healed, reconfigured, and recycled (Fig. 5a,b) [116, 117]. A liquid metal is not necessary to restore electrical conductivity, as demonstrated for a self-healing composite that incorporated Ag nanowires and CNTs. The composite healed at room
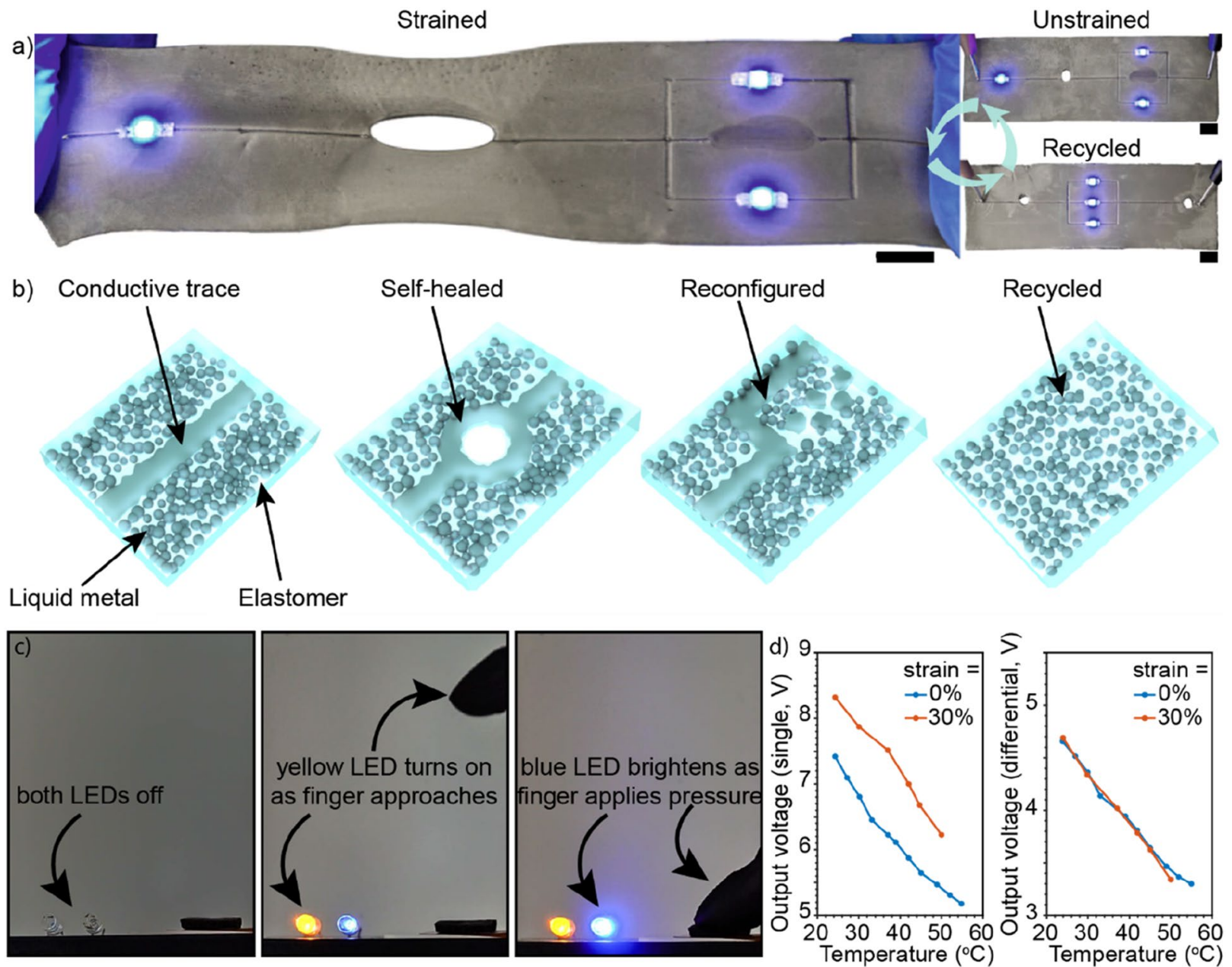

Figure 5: (a) A liquid metal composite using a self-healing matrix can be stretched and recycled, as shown in these photographs of circuits formed using the composites. The scale bars represent $10 \mathrm{~mm}$. Reproduced with permission from Ref. 115. Copyright 2021, Springer Nature. (b) The composite can form conductive or insulating regions based on post-processing (i.e., mechanical sintering). The composite is also capable of electrical self-healing, reconfiguration, and recycling, as shown in these illustrations. Reproduced with permission from Ref. 115. Copyright 2021, Springer Nature. (c) A selfhealing foam that uses multiple electrodes is capable of proximity sensing along with pressure sensing, as shown in these sequential photographs of the composite sensing. Ref. 118. Copyright 2020, Springer Nature (d) Polymer semiconductors can be used for organic field-effect transistors, which can assist with sensing. When reading a single output voltage, the temperature and strain signals can be convoluted, as shown in the plot of voltage vs. temperature on the left. For example, an output voltage of around $7 \mathrm{~V}$ could mean that the composite is unstrained at $28^{\circ} \mathrm{C}$ or is stretched at $30 \%$ strain at $42^{\circ} \mathrm{C}$. When using the differential output voltage of two transistors, strain-independent temperature sensing is possible, as shown in the plot of voltage vs. temperature on the right. Data taken from Ref. 130. 
temperature; however, complete healing required $>3 \mathrm{~h}$, which may be useful still depending on the application. The composite was integrated in an electronic device that interfaced with human skin and could indicate vital signs through electroluminescence [118]. Continued development of self-healing of multiple properties will be useful for soft materials, especially for healing that does not require intervention or sensing.

Self-healing materials have often been touted as suitable for electronic skin. Biological skin not only is self-healing but also can sense the direction of an applied force (e.g., shear vs. compression) and can detect precontact events. In many embodiments of electronic skin, a two-dimensional electrode contacts the electronic skin for sensing purposes. A recent report outlined the use of a self-healing foam filled with nickel microparticles that used three-dimensional electrodes instead of planar electrodes. These electrodes were wires that were embedded into the foam. The composite could measure either capacitive or resistive changes. Shear force and direction could be identified by monitoring the resistance between electrodes. A reference electrode was chosen, and the change in resistance between all other electrodes was monitored upon application of a shear force. The increase in resistance was greater for the electrode in the direction of shear. Detection of proximity using capacitive sensing was also possible while compression increased the resistance. Thus, a finger that approached to the material could be distinguished from a material that was pressing the material (Fig. 5c) [119]. This sensing was done simultaneously in a single material and is another example of how material properties can facilitate sensing and categorization.

\section{Transient polymer composites}

Transient materials work counter to self-healing materials. Transient polymers degrade in response to a stimulus in a manner that can be controlled. Transient materials can be used in intelligent machines that can eliminate components that are no longer needed, depending on changes in the environment. These materials rely on metastable polymers or polymers that depolymerize in the presence of specific stimuli $[120,121]$. Intelligence in transient materials could be borne out through a loss of function or by unrestricting a functionality. For example, an actuator that functions as a fin for swimming could be restricted by a transient material. Exposure to water could trigger depolymerization, and the fin could then move so that the soft machine can swim. Alternatively, a transient material could allow responsivity that allows the machine to deliver a payload when exposed to an appropriate stimulus without intervention by a processor or controller.

Poly(phthalaldehyde) (PPHA) is a common transient polymer. Above $-43{ }^{\circ} \mathrm{C}$, depolymerization is thermodynamically favorable, and stability at room temperature was improved from a few days at room temperature to at least several months through cyclization [122]. Using cyclic PPHA, transient fibers that could be used for wearable electronics were fabricated. Pyrene was included in the matrix as a photoabsorber, and a photoacid generator was included to facilitate depolymerization. In sunlight, these fibers disintegrated within $50 \mathrm{~s}$ [123]. When considering transient electronics, all components may need to be completely disintegrable. By using a disintegrable polymer semiconductor with iron electrodes, flexible circuits that were completely transient have been fabricated [124]. A challenge for transient polymers and related composites is to control when degradation occurs, which can be managed in carefully designed systems (e.g., by using materials that are triggered by different stimuli) [125].

\section{Polymer semiconductors}

Polymer conductors and semiconductors will likely be important components of multimaterial, multifunctional machines. These materials are more compliant than traditional electronic materials like metals and silicon, and performance metrics continue to improve as researchers develop an understanding of structure-property relationships [126]. Composites that incorporate a polymer semiconductor and a stretchable polymer have been used to improve stretchability $[127,128]$. To achieve stretchability, polymer semiconductors organize into nanostructures that are dispersed in a solution-processable elastomer. $\mathrm{Xu}$ et al. used these semiconductor composites to create organic field-effect transistors (OFETs). These OFETs maintained a carrier mobility of $1.1 \mathrm{~cm}^{2} \mathrm{~V}^{-1} \mathrm{~s}^{-1}$ when stretched up to $100 \%$ strain. An OFET was integrated into a circuit with an LED and adhered to a human hand to show that electrical power could be maintained during normal motion [129]. A model was developed to describe current-voltage characteristics of OFETs as a function of strain. This model highlights the potential to leverage physical intelligence in these OFETs: the output current either decreases or increases depending on the direction of the applied strain (parallel vs. perpendicular to the direction of the electric field between the source and drain electrodes) [130]. By monitoring the change in current, a single OFET could assist with proprioception by indicating the direction that a machine is being stretched.

More recently, stretchable OFETs were incorporated into an integrated circuit that could measure temperature. The effects of strain and temperature on the output voltage were deconvoluted by using a pair of transistors and monitoring the changes in threshold voltage due to strain. Whether the circuit was strained at $0 \%$ or $30 \%$ strain, the temperature readout was accurate within $1^{\circ} \mathrm{C}$ (Fig. 5d) [131]. A prototype of a wearable temperature sensor was fabricated [132]. This use of soft materials for integrated circuits solves a challenge that other soft 
sensors may face. This challenge involves the ability to categorize and deconvolute signals, which is necessary for soft machines. While physical intelligence will assist in computation, it may not eliminate its use in soft machines. Thus, traditional logic circuits using compliant materials like OFETs will be important to couple with soft materials that exhibit physical intelligence.

\section{Summary and future directions}

The development of soft and flexible materials that exhibit multifunctionality has relied heavily on advancements in polymer chemistry and processing. We have covered a small subset of novel functionalities that have emerged as polymer chemists continue to develop structure-property relationships. Physical intelligence is not limited to the materials that have been discussed here. Emerging synthetic routes will advance composites that contain biomedical polymers, optoelectronic polymers, polymer membranes for separation, covalent organic frameworks, and other stimuli-responsive polymers not discussed here [133, 134]. The future of physical intelligence-and of soft machineswill depend on the advancements in polymer chemistry.

\section{Outlook}

The interaction between a soft machine and its environment starts with the materials that comprise the machine. Therefore, materials in these machines should be capable of sensing, actuation, computation, and communication if researchers want to maximize performance and versatility [135].

This review focused on advances in developing functional polymers and polymer composites that enabled physical intelligence. A boundless number of polymers and copolymers with different properties can be synthesized when considering the number of compositional and configurational substitutions in the chemical structure. The choice of filler (or multiple fillers) and processing conditions multiplies the number of composites that can be fabricated. An Edisonian or phenomenological approach to new material development can be useful as physical intelligence is initially explored; however, research in physical intelligence should also continue to answer fundamental questions that are important to materials science.

\section{Interfaces of disparate materials}

The interface between two dissimilar materials that form a composite remains important for future work. Mismatches in properties like surface energy and modulus can be studied and overcome to maximize performance by chemically modifying interfaces or by tuning processing [136-138]. Alternatively, a soft matrix can incorporate a liquid filler that imparts the desired properties without mechanical mismatch as long as the interface of the liquid filler is controlled $[139,140]$. By studying composites with liquid fillers, new understandings of the effects of surface tension on mechanical properties led to the development of new inclusion theories [140, 141]. The interphase, which refers to the structure that connects the matrix and filler beyond the absorption layer and is important for fiber-reinforced composites, is also important to understand for functional composites with both liquid and solid inclusions. It is important to note that for high filler content and small particle size, an interphase that is $1 \mathrm{~nm}$ thick can constitute a meaningful proportion of the composite [142].

Material interfaces are important when considering multiple polymers and fillers (i.e., $>2$ components) in a single composite. The use of multiple components can introduce new functionalities like simultaneous heating and actuation of a magnetic composite that changes shape [143] or dynamic color change that is activated by pressure or changes in current [144]. Rather than introducing new functionalities, multiple components can improve functionalities relative to a single component. When combined with liquid metal, composites with Ag fillers had higher conductance at large strain than composites without liquid metal [145]. If properties like electrical conductivity are limited by interparticle interactions, then the interactions between multiple fillers will be important to understand. Multiple components could also refer to more than one polymeric material. Semisolids (e.g., gels) and other functional soft matter are underexplored as composite fillers relative to rigid composite materials. An understanding of how inclusion theory extends to semisolids is a logical step forward. Further work may consider how interfaces and interphases are influenced by covalent and/or non-covalent interactions, how the gradient of an interphase can be controlled, and how these interactions influence functionality.

\section{Additive manufacturing}

Material interfaces are also important as multimaterial printing becomes more widespread. The printing of actuators can be augmented by simultaneously printing sensors, for example [146]. In the case of a sensor, any electrodes, wires, stimuli-responsive materials, and dielectric materials required for sensing will need to be printed. The rheological properties, curing, and adhesion of these disparate materials need to be controlled for maximum performance, which can be a challenging engineering problem.

Other advances in additive manufacturing will also improve researchers' abilities to produce materials with physical intelligence. Printing that enables shape, property, or functionality that evolves over time (termed 4D printing) will allow for the creation of materials with new functionalities [147]. Within this subset of additive manufacturing, there are important aspects of polymer chemistry and fluid dynamics that require further 
understanding. In addition, there are interesting problems related to predicting the time evolution of the targeted shape/ property/functionality, especially in unstructured environments. These principles will dictate the functionality and physical intelligence that new materials possess.

\section{Self- and directed assembly}

By controlling the morphology of fillers in a composite, it may be possible to control properties that bring about physical intelligence. As discussed, the assembly of composite fillers can be controlled during processing (e.g., by alignment) [59]. Other processing techniques like additive manufacturing have been tuned to direct assembly. For example, fibrous fillers in an epoxy ink were printed by direct ink writing. Extrusion imposed a shear field on the fibers, aligning them during printing. The alignment introduced anisotropic mechanical properties for individual strands, and a printed structure could intentionally localize stress concentrations [148]. Directed assembly can be straightforward; sedimentation of fillers can create encapsulation layers for printed conductors [149], can introduce anisotropy in actuation [150], or can facilitate processing while retaining functionality [151]. Self-assembly requires an understanding of interface interactions and kinetics of processing/synthesis. Interactions that dictate the morphology between the filler and matrix are important as is the composite's architecture, which influences how the composite interacts with its environment. Structures that can fold and unfold, as examples of architectures that modify interactions with the environment, are important to consider.

Fishing wire provides a simple example of how the morphology of a material dictates its properties. When twisted into a tight coil and processed with carbon nanotubes, fishing wire can function as an artificial muscle that relies on thermal expansion and is electrothermally driven [152]. Thus, assembly across multiple length scales should be considered. In nature and in manmade objects, this so-called structural hierarchy is important for functionality, and the interplay between chemistry, processing, and morphology will be important for the progress of multifunctional materials for soft machines.

\section{Multi-input, multi-output response}

A common theme in this review involves the ability of materials to detect and differentiate between multiple signals. Many materials can respond to multiple environmental stimuli. For example, a conductive composite that is used for wearable electronics can be used to track motion. The conductivity of the composite changes when the wearer runs or walks. However, changes in conductivity that occur during running vs. walking can overlap, and the composite is unable to differentiate. One way that has been discussed to distinguish between signals involves the device architecture, where the electrical signal between multiple electrodes can be used to sense directionality [119]. Another way to decouple multiple stimuli that was discussed integrates logic devices (i.e., organic field-effect transistors) into a soft material, which was effective. Alternatively, multiplicity in sensing could be incorporated into devices. For a device that measures physiological changes, measurements of resistance over time are as important as the absolute change in resistance. For example, a device that could sense coughing, swallowing, and deep breathing [153] could distinguish these events by monitoring and filtering events deemed "healthy" or "recurring and ordinary." This filtering would also require improvements in data processing.

Future work should also consider leveraging non-linear or non-ideal responses of stretchable materials. For composites with rigid fillers, the electromechanical coupling often follows a response that deviates from the expected dependence as dictated by Pouillet's Law. This deviation can occur due to changes in particle-particle and particle-matrix interactions. In many cases, these materials are touted as having high gage factors since the change in resistance at large strain is much greater than expected. However, a gage factor is not particularly useful if it attempts to encapsulate the entire range of resistance vs. strain for a composite that displays highly non-linear electromechanical coupling. It will be more useful to consider more localized changes in resistance with strain and to capitalize on non-linearities for sensing.

Another option for multi-input, multi-output response is to couple the response with multiple-phase transitions. In shapememory polymers (SMPs), multiple shapes can be programmed when a phase transition occurs across a broad temperature range [50]. For a SMP with a broad melting transition, there are innumerable crystallites each with a slightly different local environment that changes the melting temperature. In theory, each crystallite could be accessed to sense, respond, or store information. The question that would need to be answered is, how is this information accessed by a soft machine so that it is useful? The filler can also introduce new phase transitions that can allow the composite to respond to stimuli. A potentially useful example of how the phase transitions of fillers can be used involves liquid metals. Liquid metals that contain gallium, bismuth, indium, and tin are most often used at their eutectic point. However, the entire phase space could be leveraged for multiresponsivity. Additionally, liquid metals of various compositions are known to exhibit undercooling, where the liquid does not solidify until well below its freezing temperature [154-156]. Polydispersity in liquid metal droplets could result in multiple-phase transitions, which would be useful for multiresponsivity [29]. Overall, materials that can exhibit multi-input, multi-output responsivity will be useful but will require new modes to read and understand the response. 
Soft materials can be difficult to model as they are continuously deformable, have infinite degrees of freedom, and can behave in manners that are subject to stochasticity or statistical variations. Machine learning is well suited to assist with these kinds of problems [157]. For example, a foam that contains magnetic particles was used for sensing of force and position. The sensor relied on the change in local concentration and position of the magnetic particles as detected by a single magnetometer. A neural network allowed for location and force estimation for sensors as large as $40 \mathrm{~mm}^{2}[158,159]$. A priori models of sensor or actuator dynamics based on first principles are not well suited to predicting these responses. Therefore, empirical modeling and machine learning will be useful tools for developing materials with physical intelligence.

\section{Conclusion}

Soft machines will require multifunctional materials for versatility and robustness. In many of these materials and machines, physical and/or embodied intelligence will enable new functionalities and reduce computational load. As outlined in this review, materials are already capable of multifunctionality that epitomizes physical intelligence. However, many reported examples of physical intelligence may not readily transfer outside of laboratory environment to a real-world environment that is unstructured, and so future work should consider this challenge.

There are some principles of embodied intelligence [2] that can also be useful to consider when targeting milestones for new materials with physical intelligence. (i) Intelligent systems are capable of categorization of stimuli. How can materials categorize and respond to/communicate categorization? (ii) Intelligent systems can learn and predict. Future work may focus on new materials that anticipate their environment and modify their properties to accommodate stimuli. (iii) Intelligent systems have built-in attractor states, scaffolding, and redundancy. Materials with physical intelligence should respond to their environment reliably, cheaply (e.g., energetically or computationally), and without failure in an unpredictable environment. These materials can reduce computational load by changing states without changing input signals and by relying on the properties of the materials rather than sensors. Developments in the areas discussed in this review will allow for new materials that drive soft machines-machines that can explore, protect, transform, transport, and partner with humankind [160].

\section{Acknowledgments}

This work was partially supported by the U.S. Department of Energy by Lawrence Livermore National Laboratory under contract DE-AC52-07NA27344. The authors declare no further conflict of interest.

\section{Open Access}

This article is licensed under a Creative Commons Attribution 4.0 International License, which permits use, sharing, adaptation, distribution and reproduction in any medium or format, as long as you give appropriate credit to the original author(s) and the source, provide a link to the Creative Commons licence, and indicate if changes were made. The images or other third party material in this article are included in the article's Creative Commons licence, unless indicated otherwise in a credit line to the material. If material is not included in the article's Creative Commons licence and your intended use is not permitted by statutory regulation or exceeds the permitted use, you will need to obtain permission directly from the copyright holder. To view a copy of this licence, visit http://creativecommons.org/ licenses/by/4.0/.

\section{References}

1. M. Sitti, Physical intelligence as a new paradigm. Extreme Mech. Lett. 46, 101340 (2021). https://doi.org/10.1016/j.eml. 2021.101340

2. R. Pfeifer, J. Bongard, How the Body Shapes the Way We Think: A New View of Intelligence; A Bradford Book (MIT Press, Cambridge, MA, 2007)

3. K. Bhattacharya, R.D. James, The material is the machine. Science 307(5706), 53-54 (2005). https://doi.org/10.1126/ science. 1100892

4. J.M. McCracken, B.R. Donovan, T.J. White, Materials as machines. Adv. Mater. 32(20), 1906564 (2020). https://doi. org/10.1002/adma.201906564

5. E. Brown, N. Rodenberg, J. Amend, A. Mozeika, E. Steltz, M.R. Zakin, H. Lipson, H.M. Jaeger, Universal robotic gripper based on the jamming of granular material. PNAS 107(44), 18809-18814 (2010). https://doi.org/10.1073/pnas.10032 50107

6. E.W. Hawkes, L.H. Blumenschein, J.D. Greer, A.M. Okamura, A soft robot that navigates its environment through growth. Sci. Robot. (2017). https://doi.org/10.1126/scirobotics.aan3028

7. M. Ikeuchi, Y. Ogawa, A. Iwase, K. Sugimoto, Plant regeneration: cellular origins and molecular mechanisms. Development 143(9), 1442-1451 (2016). https://doi.org/10.1242/dev.134668

8. S. Schara, R. Blau, D.C. Church, J.K. Pokorski, D.J. Lipomi, Polymer chemistry for haptics, soft robotics, and humanmachine interfaces. Adv. Funct. Mater. (2008). https://doi. org/10.1002/adfm.202008375

9. D.P. Nair, M. Podgórski, S. Chatani, T. Gong, W. Xi, C.R. Fenoli, C.N. Bowman, The Thiol-Michael addition click reaction: a powerful and widely used tool in materials chemistry. Chem. Mater. 26(1), 724-744 (2014). https://doi.org/10.1021/ $\mathrm{cm} 402180 \mathrm{t}$ 
10. B.D. Mather, K. Viswanathan, K.M. Miller, T.E. Long, Michael addition reactions in macromolecular design for emerging technologies. Prog. Polym. Sci. 31(5), 487-531 (2006). https:// doi.org/10.1016/j.progpolymsci.2006.03.001

11. M. Warner, E.M. Terentjev, Liquid Crystal Elastomers (OUP, Oxford, 2007)

12. H. Aharoni, Y. Xia, X. Zhang, R.D. Kamien, S. Yang, Universal inverse design of surfaces with thin nematic elastomer sheets. PNAS 115(28), 7206-7211 (2018). https://doi.org/10.1073/ pnas. 1804702115

13. M. Barnes, R. Verduzco, Direct shape programming of liquid crystal elastomers. Soft Matter 15(5), 870-879 (2019). https:// doi.org/10.1039/C8SM02174K

14. C.P. Ambulo, J.J. Burroughs, J.M. Boothby, H. Kim, M.R. Shankar, T.H. Ware, Four-dimensional printing of liquid crystal elastomers. ACS Appl. Mater. Interfaces 9(42), 37332-37339 (2017). https://doi.org/10.1021/acsami.7b11851

15. M. López-Valdeolivas, D. Liu, D.J. Broer, C. Sánchez-Somolinos, 4D Printed actuators with soft-robotic functions. Macromol. Rapid Commun. 39(5), 1700710 (2018). https://doi.org/10. 1002/marc.201700710

16. A. Kotikian, R.L. Truby, J.W. Boley, T.J. White, J.A. Lewis, 3D printing of liquid crystal elastomeric actuators with spatially programed nematic order. Adv. Mater. 30(10), 1706164 (2018). https://doi.org/10.1002/adma.201706164

17. S. Schuhladen, F. Preller, R. Rix, S. Petsch, R. Zentel, H. Zappe, Iris-like tunable aperture employing liquid-crystal elastomers. Adv. Mater. 26(42), 7247-7251 (2014). https://doi.org/10.1002/ adma.201402878

18. Y. Yao, J.T. Waters, A.V. Shneidman, J. Cui, X. Wang, N.K. Mandsberg, S. Li, A.C. Balazs, J. Aizenberg, Multiresponsive polymeric microstructures with encoded predetermined and self-regulated deformability. PNAS 115(51), 12950-12955 (2018). https://doi.org/10.1073/pnas.1811823115

19. M. Tabrizi, T.H. Ware, M.R. Shankar, Voxelated molecular patterning in three-dimensional freeforms. ACS Appl. Mater. Interfaces 11(31), 28236-28245 (2019). https://doi.org/10.1021/ acsami.9b04480

20. H. Zeng, O.M. Wani, P. Wasylczyk, A. Priimagi, Light-driven, caterpillar-inspired miniature inching robot. Macromol. Rapid Commun. 39(1), 1700224 (2018). https://doi.org/10.1002/marc. 201700224

21. H. Shahsavan, A. Aghakhani, H. Zeng, Y. Guo, Z.S. Davidson, A. Priimagi, M. Sitti, Bioinspired underwater locomotion of light-driven liquid crystal gels. PNAS 117(10), 5125-5133 (2020). https://doi.org/10.1073/pnas.1917952117

22. M. Camacho-Lopez, H. Finkelmann, P. Palffy-Muhoray, M. Shelley, Fast liquid-crystal elastomer swims into the dark. Nature Mater 3(5), 307-310 (2004). https://doi.org/10.1038/ nmat1118
23. O.M. Wani, H. Zeng, A. Priimagi, A light-driven artificial flytrap. Nat Commun. 8(1), 15546 (2017). https://doi.org/10.1038/ ncomms 15546

24. X. Huang, M. Ford, Z.J. Patterson, M. Zarepoor, C. Pan, C. Majidi, Shape memory materials for electrically-powered soft machines. J. Mater. Chem. B 8(21), 4539-4551 (2020). https:// doi.org/10.1039/D0TB00392A

25. Q. He, Z. Wang, Y. Wang, A. Minori, M.T. Tolley, S. Cai, Electrically controlled liquid crystal elastomer-based soft tubular actuator with multimodal actuation. Sci. Adv. 5(10), eaax5746 (2019). https://doi.org/10.1126/sciadv.aax5746

26. M.O. Saed, C.P. Ambulo, H. Kim, R. De, V. Raval, K. Searles, D.A. Siddiqui, J.M.O. Cue, M.C. Stefan, M.R. Shankar, T.H. Ware, Molecularly-engineered, 4D-printed liquid crystal elastomer actuators. Adv. Func. Mater. 29(3), 1806412 (2019). https:// doi.org/10.1002/adfm.201806412

27. A. Agrawal, H. Chen, H. Kim, B. Zhu, O. Adetiba, A. Miranda, A. Cristian Chipara, P.M. Ajayan, J.G. Jacot, R. Verduzco, Electromechanically responsive liquid crystal elastomer nanocomposites for active cell culture. ACS Macro Lett. 5(12), 1386-1390 (2016). https://doi.org/10.1021/acsma crolett.6b00554

28. M.J. Ford, C.P. Ambulo, T.A. Kent, E.J. Markvicka, C. Pan, J. Malen, T.H. Ware, C. Majidi, A Multifunctional ShapeMorphing Elastomer With Liquid Metal Inclusions. PNAS 116(43), 21438-21444 (2019). https://doi.org/10.1073/pnas. 1911021116

29. M.J. Ford, M. Palaniswamy, C.P. Ambulo, T.H. Ware, C. Majidi, Size of liquid metal particles influences actuation properties of a liquid crystal elastomer composite. Soft Matter 16(25), 5878-5885 (2020). https://doi.org/10.1039/D0SM00278J

30. J.W. Boley, E.L. White, R.K. Kramer, Mechanically sintered gallium-indium nanoparticles. Adv. Mater. 27(14), 2355-2360 (2015). https://doi.org/10.1002/adma.201404790

31. E.J. Markvicka, M.D. Bartlett, X. Huang, C. Majidi, An autonomously electrically self-healing liquid metal-elastomer composite for robust soft-matter robotics and electronics. Nat. Mater. 17(7), 618-624 (2018). https://doi.org/10.1038/ s41563-018-0084-7

32. E.J. Markvicka, R. Tutika, M.D. Bartlett, C. Majidi, Soft electronic skin for multi-site damage detection and localization. Adv. Func. Mater. 29(29), 1900160 (2019). https://doi.org/10. 1002/adfm.201900160

33. S. Zhu, J.-H. So, R. Mays, S. Desai, W.R. Barnes, B. Pourdeyhimi, M.D. Dickey, Ultrastretchable fibers with metallic conductivity using a liquid metal alloy core. Adv. Func. Mater. 23(18), 2308-2314 (2013). https://doi.org/10.1002/adfm.20120 2405

34. T.A. Kent, M.J. Ford, E.J. Markvicka, C. Majidi, Soft actuators using liquid crystal elastomers with encapsulated liquid metal 
joule heaters. Multifunct. Mater. 3(2), 025003 (2020). https:// doi.org/10.1088/2399-7532/ab835c

35. A. Kotikian, J.M. Morales, A. Lu, J. Mueller, Z.S. Davidson, J.W. Boley, J.A. Lewis, Innervated, self-sensing liquid crystal elastomer actuators with closed loop control. Adv. Mater. (2021). https://doi.org/10.1002/adma.202101814

36. B. Ma, C. Xu, L. Cui, C. Zhao, H. Liu, Magnetic printing of liquid metal for perceptive soft actuators with embodied intelligence. ACS Appl. Mater. Interfaces 13(4), 5574-5582 (2021). https://doi.org/10.1021/acsami.0c20418

37. A. Kaiser, M. Winkler, S. Krause, H. Finkelmann, A.M. Schmidt, Magnetoactive liquid crystal elastomer nanocomposites. J. Mater. Chem. 19(4), 538-543 (2009). https://doi.org/10. 1039/B813120C

38. J.M. Haberl, A. Sánchez-Ferrer, A.M. Mihut, H. Dietsch, A.M. Hirt, R. Mezzenga, Liquid-crystalline elastomer-nanoparticle hybrids with reversible switch of magnetic memory. Adv. Mater. 25(12), 1787-1791 (2013). https://doi.org/10.1002/adma.20120 4406

39. J. Zhang, Y. Guo, W. Hu, R.H. Soon, Z.S. Davidson, M. Sitti, Liquid crystal elastomer-based magnetic composite films for reconfigurable shape-morphing soft miniature machines. Adv. Mater. 33(8), 2006191 (2021). https://doi.org/10.1002/adma. 202006191

40. G. Wu, Y. Jiang, D. Xu, H. Tang, X. Liang, G. Li, Thermoresponsive inverse opal films fabricated with liquid-crystal elastomers and nematic liquid crystals. Langmuir 27(4), 1505-1509 (2011). https://doi.org/10.1021/la1037124

41. D. Mistry, M. Nikkhou, T. Raistrick, M. Hussain, E.I.L. Jull, D.L. Baker, H.F. Gleeson, Isotropic liquid crystal elastomers as exceptional photoelastic strain sensors. Macromolecules 53(10), 3709-3718 (2020). https://doi.org/10.1021/acs.macromol.9b024 56

42. N.A. Traugutt, D. Mistry, C. Luo, K. Yu, Q. Ge, C.M. Yakacki, Liquid-crystal-elastomer-based dissipative structures by digital light processing 3D printing. Adv. Mater. 32(28), 2000797 (2020). https://doi.org/10.1002/adma.202000797

43. C.M. Yakacki, M. Saed, D.P. Nair, T. Gong, S.M. Reed, C.N. Bowman, Tailorable and programmable liquid-crystalline elastomers using a two-stage thiol-acrylate reaction. RSC Adv. 5(25), 18997-19001 (2015). https://doi.org/10.1039/C5RA0 1039J

44. Z. Pei, Y. Yang, Q. Chen, E.M. Terentjev, Y. Wei, Y. Ji, Mouldable liquid-crystalline elastomer actuators with exchangeable covalent bonds. Nature Mater 13(1), 36-41 (2014). https://doi. org/10.1038/nmat3812

45. H.-F. Lu, M. Wang, X.-M. Chen, B.-P. Lin, H. Yang, Interpenetrating liquid-crystal polyurethane/polyacrylate elastomer with ultrastrong mechanical property. J. Am. Chem. Soc. 141(36), 14364-14369 (2019). https://doi.org/10.1021/jacs.9b06757
46. S.W. Ula, N.A. Traugutt, R.H. Volpe, R.R. Patel, K. Yu, C.M. Yakacki, Liquid crystal elastomers: an introduction and review of emerging technologies. Liquid Cryst. Rev. 6(1), 78-107 (2018). https://doi.org/10.1080/21680396.2018.1530155

47. D. Mistry, N.A. Traugutt, K. Yu, C.M. Yakacki, Processing and reprocessing liquid crystal elastomer actuators. J. Appl. Phys. 129(13), 130901 (2021). https://doi.org/10.1063/5.0044533

48. J. Leng, H. Lu, Y. Liu, W.M. Huang, S. Du, Shape-memory polymers-a class of novel smart materials. MRS Bull. 34(11), 848-855 (2009). https://doi.org/10.1557/mrs2009.235

49. A. Lendlein, O.E.C. Gould, Reprogrammable recovery and actuation behaviour of shape-memory polymers. Nat. Rev. Mater. 4(2), 116-133 (2019). https://doi.org/10.1038/ s41578-018-0078-8

50. J. Zotzmann, M. Behl, D. Hofmann, A. Lendlein, Reversible Triple-shape effect of polymer networks containing polypentadecalactone- and poly( $\varepsilon$-caprolactone)-segments. Adv. Mater. 22(31), 3424-3429 (2010). https://doi.org/10.1002/adma.20090 4202

51. T. Chung, A. Romo-Uribe, P.T. Mather, Two-way reversible shape memory in a semicrystalline network. Macromolecules 41(1), 184-192 (2008). https://doi.org/10.1021/ma071517z

52. K. Kratz, S.A. Madbouly, W. Wagermaier, A. Lendlein, Temperature-memory polymer networks with crystallizable controlling units. Adv. Mater. 23(35), 4058-4062 (2011). https://doi.org/10. 1002/adma.201102225

53. Y. Xia, Y. He, F. Zhang, Y. Liu, J. Leng, A review of shape memory polymers and composites: mechanisms, materials, and applications. Adv. Mater. 33(6), 2000713 (2021). https://doi.org/ 10.1002/adma.202000713

54. I.S. Gunes, G.A. Jimenez, S.C. Jana, Carbonaceous fillers for shape memory actuation of polyurethane composites by resistive heating. Carbon 47(4), 981-997 (2009). https://doi.org/10. 1016/j.carbon.2008.11.053

55. L. Ma, J. Zhao, X. Wang, M. Chen, Y. Liang, Z. Wang, Z. Yu, R.C. Hedden, Effects of carbon black nanoparticles on two-way reversible shape memory in crosslinked polyethylene. Polymer 56, 490-497 (2015). https://doi.org/10.1016/j.polymer.2014.11. 036

56. J. Leng, H. Lv, Y. Liu, S. Du, Electroactivate shape-memory polymer filled with nanocarbon particles and short carbon fibers. Appl. Phys. Lett. 91(14), 144105 (2007). https://doi.org/10. $1063 / 1.2790497$

57. J.W. Cho, J.W. Kim, Y.C. Jung, N.S. Goo, Electroactive shapememory polyurethane composites incorporating carbon nanotubes. Macromol. Rapid Commun. 26(5), 412-416 (2005). https://doi.org/10.1002/marc.200400492

58. H. Lu, K. Yu, Y. Liu, J. Leng, Sensing and actuating capabilities of a shape memory polymer composite integrated with hybrid 
filler. Smart Mater. Struct. 19(6), 065014 (2010). https://doi.org/ 10.1088/0964-1726/19/6/065014

59. J.S. Leng, X. Lan, Y.J. Liu, S.Y. Du, W.M. Huang, N. Liu, S.J. Phee, Q. Yuan, Electrical conductivity of thermoresponsive shape-memory polymer with embedded micron sized Ni powder chains. Appl. Phys. Lett. 92(1), 014104 (2008). https://doi. org/10.1063/1.2829388

60. H. Wei, X. Cauchy, I.O. Navas, Y. Abderrafai, K. Chizari, U. Sundararaj, Y. Liu, J. Leng, D. Therriault, Direct 3D printing of hybrid nanofiber-based nanocomposites for highly conductive and shape memory applications. ACS Appl. Mater. Interfaces 11(27), 24523-24532 (2019). https://doi.org/10.1021/acsami. $9 \mathrm{~b} 04245$

61. A. Lendlein, H. Jiang, O. Jünger, R. Langer, Light-induced shape-memory polymers. Nature 434(7035), 879-882 (2005). https://doi.org/10.1038/nature03496

62. M. Herath, J. Epaarachchi, M. Islam, L. Fang, J. Leng, Light activated shape memory polymers and composites: a review. Eur. Polymer J. 136, 109912 (2020). https://doi.org/10.1016/j.eurpo lymj.2020.109912

63. F. Ge, X. Lu, J. Xiang, X. Tong, Y. Zhao, An optical actuator based on gold-nanoparticle-containing temperature-memory semicrystalline polymers. Angew. Chem. 129(22), 6222-6226 (2017). https://doi.org/10.1002/ange.201612164

64. Y. Liu, J.K. Boyles, J. Genzer, M.D. Dickey, Self-folding of polymer sheets using local light absorption. Soft Matter 8(6), 1764-1769 (2012). https://doi.org/10.1039/C1SM06564E

65. X. Wang, X. Guo, J. Ye, N. Zheng, P. Kohli, D. Choi, Y. Zhang, Z. Xie, Q. Zhang, H. Luan, K. Nan, B.H. Kim, Y. Xu, X. Shan, W. Bai, R. Sun, Z. Wang, H. Jang, F. Zhang, Y. Ma, Z. Xu, X. Feng, T. Xie, Y. Huang, Y. Zhang, J.A. Rogers, Freestanding 3D mesostructures, functional devices, and shape-programmable systems based on mechanically induced assembly with shape memory polymers. Adv. Mater. 31(2), 1805615 (2019). https:// doi.org/10.1002/adma.201805615

66. M. Ha, G.S.C. Bermúdez, J.A.-C. Liu, E.S.O. Mata, B.A. Evans, J.B. Tracy, D. Makarov, Reconfigurable magnetic origami actuators with on-board sensing for guided assembly. Adv. Mater. (2008). https://doi.org/10.1002/adma.202008751

67. H. Zhang, Y. Zhao, Polymers with dual light-triggered functions of shape memory and healing using gold nanoparticles. ACS Appl. Mater. Interfaces 5(24), 13069-13075 (2013). https://doi. org/10.1021/am404087q

68. R. Mohr, K. Kratz, T. Weigel, M. Lucka-Gabor, M. Moneke, A. Lendlein, Initiation of shape-memory effect by inductive heating of magnetic nanoparticles in thermoplastic polymers. PNAS 103(10), 3540-3545 (2006)

69. Z. He, N. Satarkar, T. Xie, Y.-T. Cheng, J.Z. Hilt, Remote controlled multishape polymer nanocomposites with selective radiofrequency actuations. Adv. Mater. 23(28), 3192-3196 (2011). https://doi.org/10.1002/adma.201100646
70. T. Xie, K.A. Page, S.A. Eastman, Strain-based temperature memory effect for nafion and its molecular origins. Adv. Func. Mater. 21(11), 2057-2066 (2011). https://doi.org/10.1002/adfm. 201002579

71. K. Kratz, U. Voigt, A. Lendlein, Temperature-memory effect of copolyesterurethanes and their application potential in minimally invasive medical technologies. Adv. Func. Mater. 22(14), 3057-3065 (2012). https://doi.org/10.1002/adfm.201200211

72. P. Miaudet, A. Derré, M. Maugey, C. Zakri, P.M. Piccione, R. Inoubli, P. Poulin, Shape and temperature memory of nanocomposites with broadened glass transition. Science 318(5854), 1294-1296 (2007). https://doi.org/10.1126/science.1145593

73. J.A.-C. Liu, J.H. Gillen, S.R. Mishra, B.A. Evans, J.B. Tracy, Photothermally and magnetically controlled reconfiguration of polymer composites for soft robotics. Sci. Adv. 5(8), eaa2897 (2019). https://doi.org/10.1126/sciadv.aaw2897

74. H. Koerner, G. Price, N.A. Pearce, M. Alexander, R.A. Vaia, Remotely actuated polymer nanocomposites-stress-recovery of carbon-nanotube-filled thermoplastic elastomers. Nat. Mater. 3(2), 115-120 (2004). https://doi.org/10.1038/nmat1059

75. W.M. Huang, B. Yang, L. An, C. Li, Y.S. Chan, Water-driven programmable polyurethane shape memory polymer: demonstration and mechanism. Appl. Phys. Lett. 86(11), 114105 (2005). https://doi.org/10.1063/1.1880448

76. Y. Fang, Y. Ni, B. Choi, S.-Y. Leo, J. Gao, B. Ge, C. Taylor, V. Basile, P. Jiang, Chromogenic photonic crystals enabled by novel vapor-responsive shape-memory polymers. Adv. Mater. 27(24), 3696-3704 (2015). https://doi.org/10.1002/adma.20150 0835

77. P.R. Yadav, M.H. Rizvi, B. Kuttich, S.R. Mishra, B.S. Chapman, B.B. Lynch, T. Kraus, A.L. Oldenburg, J.B. Tracy, Plasmon-coupled gold nanoparticles in stretched shape-memory polymers for mechanical/thermal sensing. ACS Appl. Nano Mater. 4(4), 3911-3921 (2021). https://doi.org/10.1021/acsanm.1c00309

78. H. Zhang, D. Wang, N. Wu, C. Li, C. Zhu, N. Zhao, J. Xu, Recyclable, self-healing, thermadapt triple-shape memory polymers based on dual dynamic bonds. ACS Appl. Mater. Interfaces 12(8), 9833-9841 (2020). https://doi.org/10.1021/acsami.9b226 13

79. B.T. Michal, C.A. Jaye, E.J. Spencer, S.J. Rowan, Inherently photohealable and thermal shape-memory polydisulfide networks. ACS Macro Lett. 2(8), 694-699 (2013). https://doi.org/10.1021/ mz400318m

80. E.D. Rodriguez, X. Luo, P.T. Mather, Linear/network poly $(\varepsilon-$ caprolactone) blends exhibiting shape memory assisted selfhealing (SMASH). ACS Appl. Mater. Interfaces 3(2), 152-161 (2011). https://doi.org/10.1021/am101012c

81. G.I. Dzhardimalieva, B.C. Yadav, I.E. Uflyand, C.M. Oliva González, B.I. Kharisov, O.V. Kharissova, B.O. García, A review on the polymers with shape memory assisted self-healing 
properties for triboelectric nanogenerators. J. Mater. Res. (2021). https://doi.org/10.1557/s43578-021-00149-X

82. Z.-Q. Dong, Y. Cao, Q.-J. Yuan, Y.-F. Wang, J.-H. Li, B.-J. Li, S. Zhang, Redox- and glucose-induced shape-memory polymers. Macromol. Rapid Commun. 34(10), 867-872 (2013). https:// doi.org/10.1002/marc.201300084

83. L. Huang, R. Jiang, J. Wu, J. Song, H. Bai, B. Li, Q. Zhao, T. Xie, Ultrafast digital printing toward 4D shape changing materials. Adv. Mater. 29(7), 1605390 (2017). https://doi.org/10.1002/ adma.201605390

84. Y. Zhang, L. Huang, H. Song, C. Ni, J. Wu, Q. Zhao, T. Xie, 4D printing of a digital shape memory polymer with tunable high performance. ACS Appl. Mater. Interfaces 11(35), 32408-32413 (2019). https://doi.org/10.1021/acsami.9b11062

85. K. Yu, A. Ritchie, Y. Mao, M.L. Dunn, H.J. Qi, Controlled sequential shape changing components by $3 \mathrm{D}$ printing of shape memory polymer multimaterials. Proc. IUTAM 12, 193-203 (2015). https://doi.org/10.1016/j.piutam.2014.12.021

86. Y.-F. Zhang, N. Zhang, H. Hingorani, N. Ding, D. Wang, C. Yuan, B. Zhang, G. Gu, Q. Ge, Fast-response, stiffness-tunable soft actuator by hybrid multimaterial 3D printing. Adv. Func. Mater. 29(15), 1806698 (2019). https://doi.org/10.1002/adfm. 201806698

87. Q. Ge, A.H. Sakhaei, H. Lee, C.K. Dunn, N.X. Fang, M.L. Dunn, Multimaterial 4D printing with tailorable shape memory polymers. Sci Rep 6(1), 31110 (2016). https://doi.org/10.1038/srep3 1110

88. T. Chen, O.R. Bilal, K. Shea, C. Daraio, Harnessing bistability for directional propulsion of soft, untethered robots. PNAS 115(22), 5698-5702 (2018)

89. Z. Jiang, B. Diggle, I.C.G. Shackleford, L.A. Connal, Tough, self-healing hydrogels capable of ultrafast shape changing. Adv. Mater. 31(48), 1904956 (2019). https://doi.org/10.1002/adma. 201904956

90. H.-R. Lee, C.-C. Kim, J.-Y. Sun, Stretchable ionics-a promising candidate for upcoming wearable devices. Adv. Mater. 30(42), 1704403 (2018). https://doi.org/10.1002/adma.201704403

91. H. Yuk, T. Zhang, S. Lin, G.A. Parada, X. Zhao, Tough bonding of hydrogels to diverse non-porous surfaces. Nat. Mater. 15(2), 190-196 (2016). https://doi.org/10.1038/nmat4463

92. X. Zhang, X. Yang, Q. Dai, Y. Zhang, H. Pan, C. Yu, Q. Feng, S. Zhu, H. Dong, X. Cao, Tough thermoplastic hydrogels with re-processability and recyclability for strain sensors. J. Mater. Chem. B 9(1), 176-186 (2021). https://doi.org/10.1039/D0TB0 2049D

93. Z. Liu, Y. Faraj, X.-J. Ju, W. Wang, R. Xie, L.-Y. Chu, Nanocomposite smart hydrogels with improved responsiveness and mechanical properties: a mini review. J. Polym. Sci. B 56(19), 1306-1313 (2018). https://doi.org/10.1002/polb.24723

94. Amended final report on the safety assessment of polyacrylamide and acrylamide residues in cosmetics1. Int J Toxicol,
24(2), 21-50 (2005). https://doi.org/10.1080/109158105909538 42.

95. K.Y. Lee, D.J. Mooney, Hydrogels for tissue engineering. Chem. Rev. 101(7), 1869-1880 (2001). https://doi.org/10.1021/cr000 $108 \mathrm{x}$

96. Y.S. Zhang, A. Khademhosseini, Advances in engineering hydrogels. Science (2017). https://doi.org/10.1126/science.aaf36 27

97. J.-Y. Sun, X. Zhao, W.R.K. Illeperuma, O. Chaudhuri, K.H. Oh, D.J. Mooney, J.J. Vlassak, Z. Suo, Highly stretchable and tough hydrogels. Nature 489(7414), 133-136 (2012). https://doi.org/ 10.1038 /nature11409

98. X. Sun, F. Yao, J. Li, Nanocomposite hydrogel-based strain and pressure sensors: a review. J. Mater. Chem. A 8(36), 1860518623 (2020). https://doi.org/10.1039/D0TA06965E

99. J. Tavakoli, Y. Tang, Hydrogel based sensors for biomedical applications: an updated review. Polymers 9(8), 364 (2017). https://doi.org/10.3390/polym9080364

100. Y. Lee, W.J. Song, J.-Y. Sun, Hydrogel soft robotics. Mater. Today Phys. 15, 100258 (2020). https://doi.org/10.1016/j.mtphys.2020. 100258

101. X. Zhang, L.M. Bellan, Composites formed from thermoresponsive polymers and conductive nanowires for transient electronic systems. ACS Appl. Mater. Interfaces 9(26), 21991-21997 (2017). https://doi.org/10.1021/acsami.7b04748

102. Y. Zhang, K. Chen, Y. Li, J. Lan, B. Yan, L. Shi, R. Ran, Highstrength, self-healable, temperature-sensitive, MXene-containing composite hydrogel as a smart compression sensor. ACS Appl. Mater. Interfaces 11(50), 47350-47357 (2019). https://doi. org/10.1021/acsami.9b16078

103. C. Larson, B. Peele, S. Li, S. Robinson, M. Totaro, L. Beccai, B. Mazzolai, R. Shepherd, Highly stretchable electroluminescent skin for optical signaling and tactile sensing. Science 351(6277), 1071-1074 (2016). https://doi.org/10.1126/science.aac5082

104. Z. Chen, Y. Chen, M.S. Hedenqvist, C. Chen, C. Cai, H. Li, H. Liu, J. Fu, Multifunctional conductive hydrogels and their applications as smart wearable devices. J. Mater. Chem. B 9(11), 2561-2583 (2021). https://doi.org/10.1039/D0TB02929G

105. Y. Ohm, C. Pan, M.J. Ford, X. Huang, J. Liao, C. Majidi, An electrically conductive silver-polyacrylamide-alginate hydrogel composite for soft electronics. Nat. Electron. 4(3), 185-192 (2021). https://doi.org/10.1038/s41928-021-00545-5

106. J.-E. Park, H.S. Kang, J. Baek, T.H. Park, S. Oh, H. Lee, M. Koo, C. Park, Rewritable, printable conducting liquid metal hydrogel. ACS Nano 13(8), 9122-9130 (2019). https://doi.org/10.1021/ acsnano.9b03405

107. Y. Zhang, B. Yang, X. Zhang, L. Xu, L. Tao, S. Li, Y. Wei, A magnetic self-healing hydrogel. Chem. Commun. 48(74), 9305-9307 (2012). https://doi.org/10.1039/C2CC34745H

108. J. Deng, H. Yuk, J. Wu, C.E. Varela, X. Chen, E.T. Roche, C.F. Guo, X. Zhao, Electrical bioadhesive interface for 
bioelectronics. Nat. Mater. 20(2), 229-236 (2021). https://doi. org/10.1038/s41563-020-00814-2

109. G. Bovone, O.Y. Dudaryeva, B. Marco-Dufort, M.W. Tibbitt, Engineering hydrogel adhesion for biomedical applications via chemical design of the junction. ACS Biomater. Sci. Eng. (2021). https://doi.org/10.1021/acsbiomaterials.0c01677

110. M. Behl, Q. Zhao, A. Lendlein, Glucose-responsive shape-memory cryogels. J. Mater. Res. 35(18), 2396-2404 (2020). https:// doi.org/10.1557/jmr.2020.204

111. Z. Li, H. Chen, B. Li, Y. Xie, X. Gong, X. Liu, H. Li, Y. Zhao, Photoresponsive luminescent polymeric hydrogels for reversible information encryption and decryption. Adv. Sci. 6(21), 1901529 (2019). https://doi.org/10.1002/advs.201901529

112. Y.J. Tan, G.J. Susanto, H.P.A. Ali, B.C.K. Tee, Progress and roadmap for intelligent self-healing materials in autonomous robotics. Adv. Mater. 33(19), 2002800 (2021). https://doi.org/10. 1002/adma.202002800

113. B.J. Adzima, C.J. Kloxin, C.N. Bowman, Externally triggered healing of a thermoreversible covalent network via self-limited hysteresis heating. Adv. Mater. 22(25), 2784-2787 (2010). https://doi.org/10.1002/adma.200904138

114. A.S. Ahmed, R.V. Ramanujan, Curie temperature controlled self-healing magnet-polymer composites. J. Mater. Res. 30(7), 946-958 (2015). https://doi.org/10.1557/jmr.2015.59

115. E. Palleau, S. Reece, S.C. Desai, M.E. Smith, M.D. Dickey, Self-healing stretchable wires for reconfigurable circuit wiring and 3D microfluidics. Adv. Mater. 25(11), 1589-1592 (2013). https://doi.org/10.1002/adma.201203921

116. R. Tutika, A.B.M.T. Haque, M.D. Bartlett, Self-healing liquid metal composite for reconfigurable and recyclable soft electronics. Commun. Mater. 2(1), 1-8 (2021). https://doi.org/10. 1038/s43246-021-00169-4

117. L. Mou, J. Qi, L. Tang, R. Dong, Y. Xia, Y. Gao, X. Jiang, Highly stretchable and biocompatible liquid metal-elastomer conductors for self-healing electronics. Small 16(51), 2005336 (2020). https://doi.org/10.1002/smll.202005336

118. D. Son, J. Kang, O. Vardoulis, Y. Kim, N. Matsuhisa, J.Y. Oh, J.W. To, J. Mun, T. Katsumata, Y. Liu, A.F. McGuire, M. Krason, F. Molina-Lopez, J. Ham, U. Kraft, Y. Lee, Y. Yun, J.B.-H. Tok, Z. Bao, An integrated self-healable electronic skin system fabricated via dynamic reconstruction of a nanostructured conducting network. Nature Nanotech 13(11), 1057-1065 (2018). https://doi.org/10.1038/s41565-018-0244-6

119. H. Guo, Y.J. Tan, G. Chen, Z. Wang, G.J. Susanto, H.H. See, Z. Yang, Z.W. Lim, L. Yang, B.C.K. Tee, Artificially innervated self-healing foams as synthetic piezo-impedance sensor skins. Nat Commun 11(1), 5747 (2020). https://doi.org/10.1038/ s41467-020-19531-0

120. H.L. Hernandez, S.-K. Kang, O.P. Lee, S.-W. Hwang, J.A. Kaitz, B. Inci, C.W. Park, S. Chung, N.R. Sottos, J.S. Moore, J.A. Rogers, S.R. White, Triggered transience of metastable poly(phthalaldehyde) for transient electronics. Adv. Mater. 26(45), 7637-7642 (2014). https://doi.org/10.1002/adma. 201403045

121. E.M. Lloyd, H. Lopez Hernandez, E.C. Feinberg, M. Yourdkhani, E.K. Zen, E.B. Mejia, N.R. Sottos, J.S. Moore, S.R. White, Fully recyclable metastable polymers and composites. Chem. Mater. 31(2), 398-406 (2019). https://doi.org/10.1021/ acs.chemmater.8b03585

122. J.A. Kaitz, C.E. Diesendruck, J.S. Moore, End group characterization of poly(phthalaldehyde): surprising discovery of a reversible, cationic macrocyclization mechanism. J. Am. Chem. Soc. 135(34), 12755-12761 (2013). https://doi.org/10. $1021 / \mathrm{ja} 405628 \mathrm{~g}$

123. S. Li, M.H. Rizvi, B.B. Lynch, J.B. Tracy, E. Ford, Flexible cyclic-poly(phthalaldehyde)/poly( $\varepsilon$-caprolactone) blend fibers with fast daylight-triggered transience. Macromol. Rapid Commun. 42(7), 2000657 (2021). https://doi.org/10.1002/ marc. 202000657

124. T. Lei, M. Guan, J. Liu, H.-C. Lin, R. Pfattner, L. Shaw, A.F. McGuire, T.-C. Huang, L. Shao, K.-T. Cheng, J.B.-H. Tok, Z. Bao, Biocompatible and totally disintegrable semiconducting polymer for ultrathin and ultralightweight transient electronics. PNAS 114(20), 5107-5112(2017)

125. C.W. Park, S.-K. Kang, H.L. Hernandez, J.A. Kaitz, D.S. Wie, J. Shin, O.P. Lee, N.R. Sottos, J.S. Moore, J.A. Rogers, S.R. White, Thermally triggered degradation of transient electronic devices. Adv. Mater. 27(25), 3783-3788 (2015). https://doi.org/10.1002/ adma.201501180

126. J.Y. Oh, S. Rondeau-Gagné, Y.-C. Chiu, A. Chortos, F. Lissel, G.J.N. Wang, B.C. Schroeder, T. Kurosawa, J. Lopez, T. Katsumata, J. Xu, C. Zhu, X. Gu, W.-G. Bae, Y. Kim, L. Jin, J.W. Chung, J.B.-H. Tok, Z. Bao, Intrinsically stretchable and healable semiconducting polymer for organic transistors. Nature 539(7629), 411-415 (2016). https://doi.org/10.1038/nature20102

127. E. Song, B. Kang, H.H. Choi, D.H. Sin, H. Lee, W.H. Lee, K. Cho, Stretchable and transparent organic semiconducting thin film with conjugated polymer nanowires embedded in an elastomeric matrix. Adv. Electron. Mater. 2(1), 1500250 (2016). https://doi.org/10.1002/aelm.201500250

128. W.K. Tatum, C.K. Luscombe, $\pi$-Conjugated polymer nanowires: advances and perspectives toward effective commercial implementation. Polym. J. 50(8), 659-669 (2018). https://doi.org/10. 1038/s41428-018-0062-6

129. J. Xu, S. Wang, G.-J.N. Wang, C. Zhu, S. Luo, L. Jin, X. Gu, S. Chen, V.R. Feig, J.W.F. To, S. Rondeau-Gagné, J. Park, B.C. Schroeder, C. Lu, J.Y. Oh, Y. Wang, Y.-H. Kim, H. Yan, R. Sinclair, D. Zhou, G. Xue, B. Murmann, C. Linder, W. Cai, J.B.-H. Tok, J.W. Chung, Z. Bao, Highly stretchable polymer semiconductor films through the nanoconfinement effect. Science 355(6320), 59-64 (2017). https://doi.org/10.1126/science.aah44 96 
130. V.G. Reynolds, S. Oh, R. Xie, M.L. Chabinyc, Model for the electro-mechanical behavior of elastic organic transistors. J. Mater. Chem. C 8(27), 9276-9285 (2020). https://doi.org/10. 1039/D0TC01181A

131. C. Zhu, H.-C. Wu, G. Nyikayaramba, Z. Bao, B. Murmann, Intrinsically stretchable temperature sensor based on organic thin-film transistors. IEEE Electron. Dev. Lett. 40(10), 1630-1633 (2019). https://doi.org/10.1109/LED.2019.2933838

132. Zhu, C., Schell, E., Kim, M., Bao, Z., Murmann, B. Wearable system design using intrinsically stretchable temperature sensor. In 2020 IEEE International Symposium on Circuits and Systems (ISCAS); 2020. https://doi.org/10.1109/ISCAS45731. 2020.9180499

133. K. Wang, K. Amin, Z. An, Z. Cai, H. Chen, H. Chen, Y. Dong, X. Feng, W. Fu, J. Gu, Y. Han, D. Hu, R. Hu, D. Huang, F. Huang, F. Huang, Y. Huang, J. Jin, X. Jin, Q. Li, T. Li, Z. Li, Z. Li, J. Liu, J. Liu, S. Liu, H. Peng, A. Qin, X. Qing, Y. Shen, J. Shi, X. Sun, B. Tong, B. Wang, H. Wang, L. Wang, S. Wang, Z. Wei, T. Xie, C. Xu, H. Xu, Z.-K. Xu, B. Yang, Y. Yu, X. Zeng, X. Zhan, G. Zhang, J. Zhang, M. Qiu Zhang, X.-Z. Zhang, X. Zhang, Y. Zhang, Y. Zhang, C. Zhao, W. Zhao, Y. Zhou, Z. Zhou, J. Zhu, X. Zhu, B. Zhong Tang, Advanced functional polymer materials. Mater. Chem. Frontiers 4(7), 1803-1915 (2020). https://doi.org/10. 1039/D0QM00025F

134. E.B. Berda, L.F. Deravi, E.J. Foster, Y. Simon, M.M. Thuo, Virtual issue: next-generation smart materials. Macromolecules 52(17), 6339-6341 (2019). https://doi.org/10.1021/acs.macro mol.9b01402

135. M.A. McEvoy, N. Correll, Materials that couple sensing, actuation, computation, and communication. Science (2015). https:// doi.org/10.1126/science.1261689

136. N. Ilyas, A. Cook, C.E. Tabor, Designing liquid metal interfaces to enable next generation flexible and reconfigurable electronics. Adv. Mater. Interfaces 4(15), 1700141 (2017). https://doi. org/10.1002/admi.201700141

137. A. Martin, C. Du, A.M. Pauls, T. Ward, M. Thuo, Polydispersitydriven printing of conformal solid metal traces on non-adhering biological surfaces. Adv. Mater. Interfaces 7(22), 2001294 (2020). https://doi.org/10.1002/admi.202001294

138. A. Martin, W. Kiarie, B. Chang, M. Thuo, Chameleon metals: autonomous nano-texturing and composition inversion on liquid metals surfaces. Angew. Chem. Int. Ed. 59(1), 352-357 (2020). https://doi.org/10.1002/anie.201912639

139. R.W. Style, R. Tutika, J.Y. Kim, M.D. Bartlett, Solid-liquid composites for soft multifunctional materials. Adv. Func. Mater. 31(1), 2005804 (2021). https://doi.org/10.1002/adfm.202005804

140. R.W. Style, R. Boltyanskiy, B. Allen, K.E. Jensen, H.P. Foote, J.S. Wettlaufer, E.R. Dufresne, Stiffening solids with liquid inclusions. Nat. Phys. 11(1), 82-87 (2015). https://doi.org/10.1038/ nphys 3181
141. C. Chiew, M.H. Malakooti, A double inclusion model for liquid metal polymer composites. Compos. Sci. Technol. 208, 108752 (2021). https://doi.org/10.1016/j.compscitech.2021.108752

142. D. Ciprari, K. Jacob, R. Tannenbaum, Characterization of polymer nanocomposite interphase and its impact on mechanical properties. Macromolecules 39(19), 6565-6573 (2006). https:// doi.org/10.1021/ma0602270

143. Q. Ze, X. Kuang, S. Wu, J. Wong, S.M. Montgomery, R. Zhang, J.M. Kovitz, F. Yang, H.J. Qi, R. Zhao, Magnetic shape memory polymers with integrated multifunctional shape manipulation. Adv. Mater. 32(4), 1906657 (2020). https://doi.org/10.1002/ adma.201906657

144. Y. Jin, Y. Lin, A. Kiani, I.D. Joshipura, M. Ge, M.D. Dickey, Materials tactile logic via innervated soft thermochromic elastomers. Nat. Commun. 10(1), 4187 (2019). https://doi.org/10. 1038/s41467-019-12161-1

145. J. Wang, G. Cai, S. Li, D. Gao, J. Xiong, P.S. Lee, Printable superelastic conductors with extreme stretchability and robust cycling endurance enabled by liquid-metal particles. Adv. Mater. 30(16), 1706157 (2018). https://doi.org/10.1002/adma. 201706157

146. A. Boydston, B. Cao, A. Nelson, R. Ono, A. Saha, J. Schwartz, C. Thrasher, Additive manufacturing with stimuli-responsive materials. J. Mater. Chem. A 6(42), 20621-20645 (2018). https:// doi.org/10.1039/C8TA07716A

147. X. Kuang, D.J. Roach, J. Wu, C.M. Hamel, Z. Ding, T. Wang, M.L. Dunn, H.J. Qi, Advances in 4D printing: materials and applications. Adv. Func. Mater. 29(2), 1805290 (2019). https:// doi.org/10.1002/adfm.201805290

148. B.G. Compton, J.A. Lewis, 3D-printing of lightweight cellular composites. Adv. Mater. 26(34), 5930-5935 (2014). https://doi. org/10.1002/adma.201401804

149. T.V. Neumann, E.G. Facchine, B. Leonardo, S. Khan, M.D. Dickey, Direct write printing of a self-encapsulating liquid metal-silicone composite. Soft Matter 16(28), 6608-6618 (2020). https://doi.org/10.1039/D0SM00803F

150. Y. Chen, Z. Chen, C. Chen, H. Ur Rehman, H. Liu, H. Li, M.S. Hedenqvist, A gradient-distributed liquid-metal hydrogel capable of tunable actuation. Chem. Eng. J. 421, 127762 (2021). https://doi.org/10.1016/j.cej.2020.127762

151. M.J. Ford, D.K. Patel, C. Pan, S. Bergbreiter, C. Majidi, Controlled assembly of liquid metal inclusions as a general approach for multifunctional composites. Adv. Mater. 32(46), 2002929 (2020). https://doi.org/10.1002/adma.202002929

152. C.S. Haines, M.D. Lima, N. Li, G.M. Spinks, J. Foroughi, J.D.W. Madden, S.H. Kim, S. Fang, M.J. de Andrade, F. Göktepe, Ö. Göktepe, S.M. Mirvakili, S. Naficy, X. Lepró, J. Oh, M.E. Kozlov, S.J. Kim, X. Xu, B.J. Swedlove, G.G. Wallace, R.H. Baughman, Artificial muscles from fishing line and sewing thread. Science 343(6173), 868-872 (2014). https://doi.org/10.1126/science. 1246906 
153. Z. Chen, J. Liu, Y. Chen, X. Zheng, H. Liu, H. Li, Multiplestimuli-responsive and cellulose conductive ionic hydrogel for smart wearable devices and thermal actuators. ACS Appl. Mater. Interfaces 13(1), 1353-1366 (2021). https://doi.org/10. 1021/acsami.0c16719

154. S. Çınar, I.D. Tevis, J. Chen, M. Thuo, Mechanical fracturing of core-shell undercooled metal particles for heat-free soldering. Sci Rep 6(1), 21864 (2016). https://doi.org/10.1038/srep21864

155. M.H. Malakooti, N. Kazem, J. Yan, C. Pan, E.J. Markvicka, K. Matyjaszewski, C. Majidi, Liquid metal supercooling for low-temperature thermoelectric wearables. Adv. Func. Mater. 29(45), 1906098 (2019). https://doi.org/10.1002/adfm.20190 6098

156. G.B. Parravicini, A. Stella, P. Ghigna, G. Spinolo, A. Migliori, F. d'Acapito, R. Kofman, Extreme undercooling (down to 90K) of liquid metal nanoparticles. Appl. Phys. Lett. 89(3), 033123

(2006). https://doi.org/10.1063/1.2221395

157. K. Chin, T. Hellebrekers, C. Majidi, Machine learning for soft robotic sensing and control. Adv. Intell. Syst. 2(6), 1900171 (2020). https://doi.org/10.1002/aisy.201900171

158. T. Hellebrekers, O. Kroemer, C. Majidi, Soft magnetic skin for continuous deformation sensing. Adv. Intell. Syst. 1(4), 1900025 (2019). https://doi.org/10.1002/aisy.201900025

159. T. Hellebrekers, N. Chang, K. Chin, M.J. Ford, O. Kroemer, C. Majidi, Soft magnetic tactile skin for continuous force and location estimation using neural networks. IEEE Robot. Automat. Lett. 5(3), 3892-3898 (2020). https://doi.org/10.1109/LRA. 2020.2983707

160. Codex. The Path to Embodied Intelligence - Anima Anandkumar. 